\title{
ICHTHYOFAUNAL DIVERSITY OF THE ADYAR WETLAND COMPLEX, ChenNAI, TAMIL NADU, SOUTHERN INDIA
}

\section{Eric Ramanujam ${ }^{1}$, K. Rema Devi $^{2} \&$ T.J. Indra ${ }^{3}$}

${ }^{1}$ Principal Investigator (Faunistics), Pitchandikulam Forest Consultants, Auroville, Tamil Nadu 605101, India ${ }^{2}$ Scientist E, ${ }^{3}$ Assistant Zoologist, Zoological Survey of India (Southern Regional Station), 130, Santhome High Road, Chennai, Tamil Nadu 600028, India

${ }^{1}$ ericramanujamowl@yahoo.com (corresponding author), ${ }^{2}$ remadevi_zsi@yahoo.com, ${ }^{3}$.jpandurangan@hotmail.com

\section{OPEN ACCESS}

\begin{abstract}
Most parts of the Adyar wetland complex-Chembarampakkam Tank, Adyar River, Adyar Estuary and Adyar backwater (including a wetland rehabilitation site)-were sampled for ichthyofaunal diversity from March 2007 to June 2011. A total of 3,732 specimens were collected and 98 taxa were identified. Twenty-two new records are reported from the estuarine reach. Forty-nine species were recorded at Chembarampakkam Tank. In the upriver stretch 42 species were recorded. In the middle stretch 25 species were encountered. In the lower stretch only five species were recorded. This lack of diversity in the lower stretch of the river can be directly linked to pollution, especially the lower reaches from Nandambakkam Bridge to Kotturpuram which exhibit anoxic conditions for most of the year. In brackish, saline and marginal waters of the estuarine reach 66 species were recorded, of which 47 occurred in the estuary proper, 34 at the point of confluence with the Bay of Bengal and 20 in the backwater which forms the creek. At the rehabilitation site, popularly referred to as the Adyar Eco Park, which is the westernmost region of the backwater, 28 species were recorded in 2011 which is a sharp increase from just four in 2007 when the area was an environmental disaster. A significant finding was Mystus cf. gulio which showed a marked morphometric difference concerning its maxillary barbels and efforts are on to discern its taxonomic status.
\end{abstract}

Keywords: Adyar Eco Park, Adyar Poonga, alien species, homogenisation, rehabilitation, translocated species, water quality, wetland complex.

DOI: http://dx.doi.org/10.11609/JoTT.o2905.5613-35 | ZooBank: urn:Isid:zoobank.org:pub:9024036E-18C6-458B-9C7C-B37EBA8F8A4D

Editor: Neelesh Dahanukar, IISER, Pune, India.

Date of publication: 26 April 2014 (online \& print)

Manuscript details: Ms \# 02905 | Received 08 August 2011 | Final received 12 March 2014 | Finally accepted 21 March 2014

Citation: Ramanujam, M.E., K.R. Devi \& T.J. Indra (2014). Ichthyofaunal diversity of the Adyar Wetland complex, Chennai, Tamil Nadu, southern India. Journal of Threatened Taxa 6(4): 5613-5635; http://dx.doi.org/10.11609/JoTT.02905.5613-35

Copyright: ( ) Ramanujam et al. 2014. Creative Commons Attribution 4.0 International License. JoTT allows unrestricted use of this article in any medium, reproduction and distribution by providing adequate credit to the authors and the source of publication.

Funding: Governmentt of Tamil Nadu and Chennai River Restoration Trust (CRRT).

Competing Interest: The authors declare no competing interests.

Author Contribution: KRD undertook the identification of all fish specimens with the help of TJI. MER collected the specimens and prepared the ms with inputs from KRD.

Author Details: M. ERIC RAMANUJAM has been a wildlife illustrator for over two decades and has a background in the advertising industry. Since 1997 he has been involved in full time conservation and has been part of a team which undertook wildlife surveys in the Kaliveli region near Puducherry, Adyar wetland complex in Chennai and the Eastern Ghats. His main sphere of interest is the natural history of the Indian Eagle Owl Bubo bengalensis. DR. K. REMA DEVI is a retired senior scientist from the Zoological Survey of India (Southern Regional Station) and an ichthyologist who has published over a hundred papers including descriptions of several new species. She obtained her DSc degree from the University of Madras in November 2012. She has served as the Regional Co-Chair of the Freshwater Fish Specialist Group (FFSG) of the IUCN for South Asia from 2009-2012. DR. T.J. INDRA is a senior scientist from the Zoological Survey of India (Southern Regional Station) and an ichthyologist who is also a specialist on scorpions. She has published several papers including descriptions of new species.

Acknowledgements: We are thankful to the Government of Tamil Nadu / Chennai Rivers Restoration Trust (CRRT) for facilitating this survey. We are also grateful to Dr. R.J. Ranjit Daniels for his valuable inputs and constructive criticism. We also wish to thank J.D. Marcus Knight for helping with this effort.

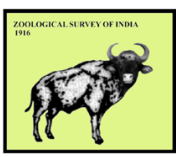




\section{INTRODUCTION}

Ichthyofaunal constituents of two areas of the Adyar wetland complex have been documented: the Adyar Estuary (Raj 1916; Panikkar \& Aiyar 1937; Anon 1950; Evangeline 1967b; Nammalwar 1982) and one source of the river at Chembarampakkam (Ragunathan 1978; Daniels \& Rajagopal 2004). These benchmarks have proved invaluable to the present analysis because it is now possible to correlate historical data to present findings.

In addition, an 'Adyar Poonga Ecological Restoration Plan', popularly referred to as the 'Master Plan', for the rehabilitation of a part of the Adyar Creek was submitted which contained information of species encountered on the proposed site (Anonymous 2007) and this was followed by a survey of parts of the Adyar Wetland complex (Ramanujam et. al. 2008, 2010). This allows for further comparative analysis and it is now possible to report on colonisation processes at the rehabilitation site.

This is a consolidated report representing data generated from 2007-2011. It aims to present findings of ichthyofaunal diversity of the Adyar Estuary, Adyar Creek, Adyar River and Chembarampakkam Tank. It also correlates historical data to present surveys of the estuarine reach. Such a comparative analysis has not been attempted for Chembarampakkam as results and analysis of a more intensive survey have already been published (Knight 2010b). Nevertheless, the results of our sampling are included in Table 1 as there are a few records that are unique to this survey.

\section{STUDY AREAS (Image 1)}

Chembarampakkam Tank $\left(1^{\circ} 0^{\prime} \mathrm{N} \& \mathbf{8 0}^{\circ} 03^{\prime} \mathrm{E}\right)$ : This is a large reservoir with a catchment of approximately $357 \mathrm{~km}^{2}$ with bunds running for at least $9 \mathrm{~km}$ around it. It offers a number of seasonal microhabitats - first there is extensive open water that is deep. Along the margins, where earth has been quarried, there are smaller temporary pools supporting dense growth of Hydrilla and Urticularia. Elsewhere, the water flows as small streams towards the sluice gates and beyond creating smaller ponds with water lilies and emergent vegetation including Cyperus and Ipomea carnea (Daniels \& Rajagopal 2004). The man-made Bangaru Canal constructed in 2004-2005 connects it via the Kortaliyar River to the Poondi Reservoir and there forth to the Krishna River. The outfall of the canal occurs at Settipalayam.

Adyar River: The river flows for about $42 \mathrm{~km}$ before joining the Bay of Bengal in Adyar. It varies in depth from approximately $0.75 \mathrm{~m}$ in its upper reaches to 0.5 $\mathrm{m}$ in its lower reaches. It discharges upto 940 million $\mathrm{cu} \mathrm{m}$ of water to the sea annually. From its origins to Tiruneermalai the water is unpolluted. A little after Tiruneermalai, the Pammal drain joins the river bringing tannery effluents and sewage. In spite of this, fishing activities are common during the rainy seasons up to Nandambakkam [the stretch of river from sources to

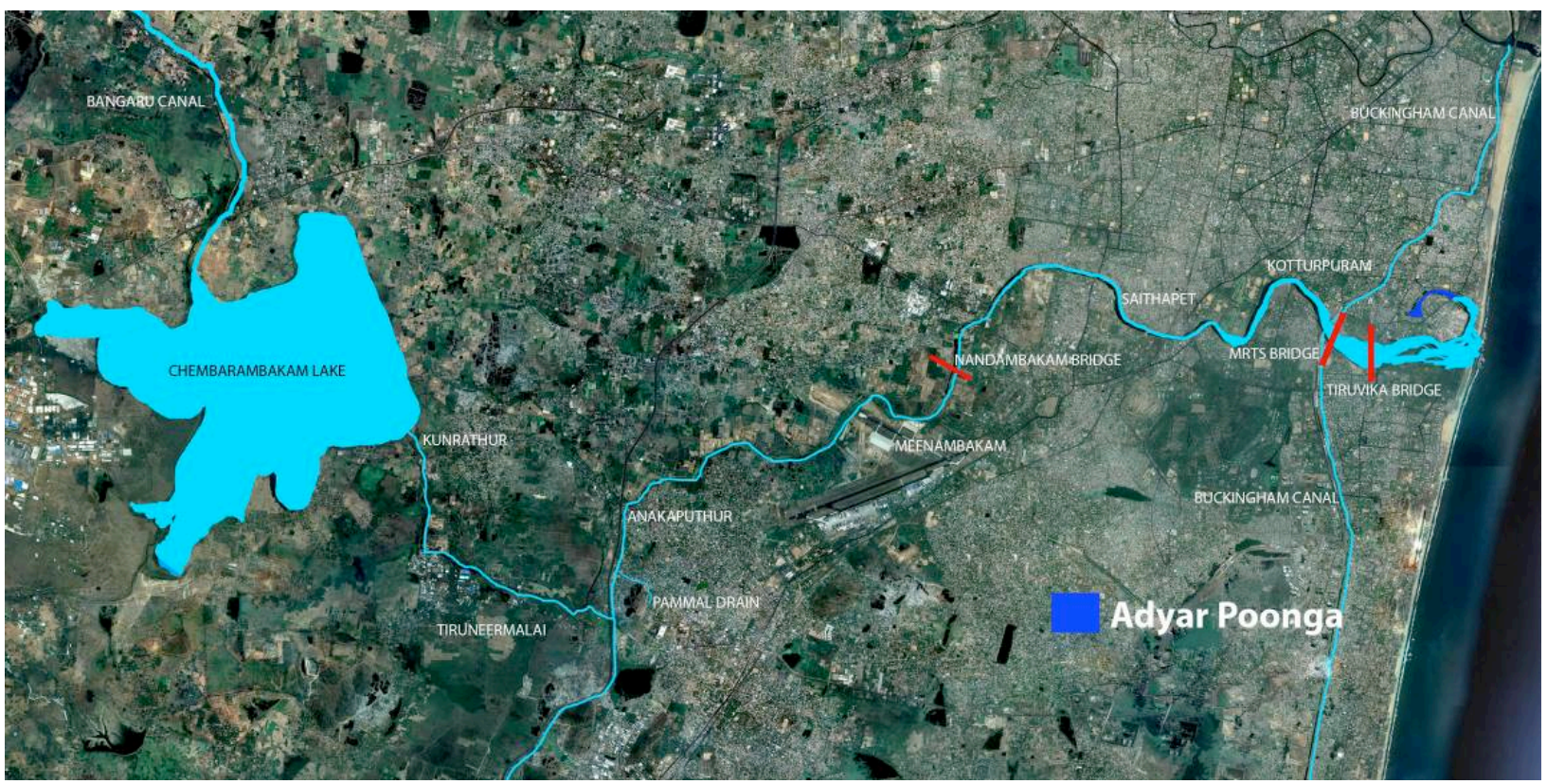

Image 1. Adyar wetland complex from Chembarampakkam tank to point of confluence with the Bay of Bengal 
Nandambakkam has been referred to as upriver in this manuscript]. The river receives a sizeable quantity of sewage and other pollutants from Chennai after reaching Nandambakkam Bridge and the water quality from here to M.R.T.S. Bridge at Kotturpuram is poor [this stretch has been referred to as downriver]. Just below the Adyar Boat Club the Buckingham Canal joins the Adyar River. This canal, built between 1806 and 1878 is a $420 \mathrm{~km}$ channel running parallel to the coast from Vijayawada on the banks of the Krishna River in Andhra Pradesh to Alamparai, near Marakkanam, in Tamil Nadu. The canal connects most of the waterways along the coast, including the Adyar.

Adyar Estuary $\left(13^{\circ} 0^{\prime} \mathrm{N} \& 8^{\circ} 16^{\prime} \mathrm{E}\right)$ : Historically, it was a typical bar-mouth estuary, i.e., the mouth remained closed for most of the year (about 9-10 months) during the dry period due to the formation of a sand bar and during the north-east monsoon (October to December) heavy freshwater flow from upriver opened the barrier to the sea. This condition was temporary and existed only during the monsoonal period. The reason for this is that the southeastern seaboard of India, also known as the Coromandel Coast, has a dissymmetric climatic regime - i.e., though the average rainfall in a year is about $1,200 \mathrm{~mm}$, the bulk of it (approximately $800 \mathrm{~mm}$ ) falls during the north-east monsoon due to depressions formed in the Bay of Bengal (Blasco \& Legris 1972; Meher-Homji, 1974-1975). Due to the shallowness of the estuary, as one approaches the point of confluence with the Bay of Bengal, a few island formations can be seen east of the Tiruvika and Elphinstone bridges. As with all estuaries worldwide, this was an 'oscillating ecosystem' and the temporal occurrence of free swimming faunal elements was at one time dictated by the opening and closing of the sand bar (Panikkar \& Aiyar 1937). There are strong indications that the entire area was well vegetated with mangroves and mangrove associates, not only on the bank of the Theosophical Society campus, but also on the islands and mudflats and records indicate that they occupied an area of about 48ha (Selvam et al. 1994).

Estuaries, the world over, are indicators of environmental health, not only because of anthropogenic activities affecting them directly, but also because all the accumulated upriver abuse is discernable here. This is especially pertinent to estuaries within metros many of the world's great cities (like London, New York and Tokyo), and Indian ones too (Calcutta, Mumbai and Chennai), developed around them because they were natural harbours. As a result, they are centres for accumulated pollution and have been dredged or filled and transformed into seaports, parks, cities and garbage dumps. Many have been obliterated and most surviving ones are endangered (Castro \& Huber 1997). The Adyar estuary is no exception. It is heavily polluted by a variety of industries, commercial establishments and direct and indirect discharge of sewage resulting in physical, chemical and biological changes. The discharge of sewage and industrial effluents is indicated by the high quantities of dissolved solids, total suspended solids, total dissolved solids, HOD, COD, ammonia and sulphates, and the concentration of heavy metals like cadmium and lead is higher than safe levels (Sivakumari et. al. 2005). The morphology of the estuary too has been tampered with. During the survey the mouth was kept open in the hope that pollutants would wash away into the sea. The vegetation is a faint shadow of its former self - only a few Avicennia marina exist on the banks of the Theosophical Society campus. The islands have been completely overrun by Prosopis juliflora and are hotbeds of antisocial activities.

Adyar Creek: The overwhelming factor here is encroachment. Encroachments from all quarters, viz.: government agencies (e.g., Ambedkar Mani Mandapapam), builders (e.g., Belisha Towers, Raja Muthia Chettiar Residential Apartments, Leela Hotel, Rani Meyyamai Apartments, Jain Sagarika) and slums at Srinavasapuram. As a result, the morphology of the wetland has been irrevocably altered and the water spread has shrunk drastically.

It is ironic that the first anthropogenic activity that intruded into the wetland concerned fish. Under the 'Second Five Year Plan' (1956-1961) the Brackish Water Fish Farm was established which was the first of its kind in Tamil Nadu (then the Madras State). The farm was 55.4 acres in extent of which 36 acres comprised of a reservoir formed by the construction of a regulator across the narrowest portion of the creek. The operation of this regulator (which consisted of three pairs of screw gearing shutters) during tidal ingress and egress facilitated the entrapping of fish, mostly during the juvenile stages. A series of nurseries, rearing ponds, stock ponds and marketing ponds covering an area of 19.4 acres was situated on the southern bank of the reservoir (Evangeline 1967a).

Since then, development based on reclamation took precedence because of the burgeoning human population associated with urbanization. The area between the seafront and creek was converted into a housing colony to accommodate displaced slum dwellers. This place of relocation, called Srinivasapuram, was begun in 19761977. Originally the housing colony consisted of LIG (Low Income Group) flats, but almost immediately hutments 
and small brick structures sprang up around them. Encroachments into the wetland were inevitable and a post tsunami count disclosed 647 huts in Srinivasapuram, of which 54 occupied the creek itself.

Development, construction and encroachment are ongoing processes and construction debris and other wastes continue to be dumped into the creek extending its banks up to $20 \mathrm{~m}$ and obliterating all mudflats. Due to this the creek has shrunk in width to only a narrow channel which hardly allows any tidal influence to the upper parts on a daily basis. In addition, a bus terminus and a memorial to Dr. Ambedkar were constructed on prime wetland habitat and more development activities based on reclamation were planned. A group of concerned citizens, members of EXNORA and Citizen Consumer and Civic Action Group (CAG), moved the court and a stay order prevented further development. In February 2008 the Government of Tamil Nadu's proposal to develop an eco-park and rehabilitate the wetland was accorded permission by the Madras High Court and the 'Adyar Poonga Ecological Restoration Plan' was put into operation. This included clearing of thousands of tonnes of rubble, stopping pollution entering the site, elimination of alien invasive Prosopis juliflora and Eichhoria crassipes, planting of mangroves and other suitable coastal vegetation, etc. Since bio-inventorisation processes were fundamental to evolve conservation strategies, faunal surveys were undertaken and this report is an outcome of such an exercise.

Period: The survey went through three phases:

1. The institutional 'Master Plan' stage was the preliminary phase that documented vertebrate diversity from March-June 2007.

2. A vertebrate diversity inventorisation process of the Adyar Wetland complex was jointly undertaken from July 2007 to October 2008 by the Zoological Survey of India (Southern Regional Station), Pitchandikulam Forest Consultants and Trust for Environment Monitoring and Action Initiating. Sampling occurred in Chembarampakkam, Adyar River, Adyar Estuary and the creek - including the rehabilitation site.

3. Updating is a continuing process. Sampling at Chembarampakkam had to be discontinued as the governmental TOR was confined to the creek and estuary alone.

This is a consolidated report of all three phases-i.e., from March 2007 to June 2011, a period of four years and three months.

\section{METHODS}

Fish were collected with the help of local fishermen who used hand-operated dragnets, seine nets, cast nets, and the hook and line. In addition, small fish that commercialists ignore were collected by the survey team using dip nets. Identification was according to established literature (Daniels 2002; Jayaram, 1981, 2006, 2010; Talwar \& Kacker 1984; Talwar \& Jhingran 1991).

\section{RESULTS AND DISCUSSION}

A total of 101 species were encountered during the survey. Forty-nine species occurred in Chembarampakkam Tank, 22 in the upriver stretch from Chembarampakkam to Nandambakkam and four in the downriver stretch from Nandambakkam to Kotturpuram [the four species were Puntius chola, Mystus cf. gulio, Oreochromis mossambica and Anabas testudineus]. The lack of fish fauna can be linked to poor water quality because in summer, even when the sandbar is opened, this stretch is reduced to a languid line of sewage and sullage. It is unreasonable to suppose that fishes do not occur in polluted water stretches-Narayanan (1980) reported seven species from polluted waters of the Cooum [for the record: Megalops cyprinoides, Muraena sp., Leiognathus sp., Terapon jarbua, Oreochromis mossambica, Liza microlepis and Mugil cephalus] and subsequently two other species were recorded in it as recently as 2007 [Oreochromis mossambica and Mystus gulio (Bai et al. 2007)]. Fiftyseven species were recorded in the estuarine reach of the Adyar, of which 49 were encountered in the estuary, 35 at the point of confluence with the Bay of Bengal and 20 in Srinivasapuram Creek. At the rehabilitation site (Adyar Poonga) 28 species have been recorded so far of which 13 species were encountered in brackish water east of the spillway and 21 in fresh water west of the spillway. For more details refer Table 1 and Fig. 1.

Prior to this survey five studies were conducted in the estuary from 1916-1982: in order of reporting, Raj (1916), Panikkar \& Aiyar (1937), Anon (1950), Evangeline (1967b), Nammalwar (1982). Including this survey 144 species have been known to occur in the estuarine reach. From a casual perusal of Fig. 2 one would be inclined to conclude that the species inventory during this survey is higher than those of 1916 (which recorded 33 species), 1937 (50 species), 1967 (41 species) and 1982 (45 species). This may not be an accurate estimate as the ambiguity concerning the area, period and intensity of surveys may have created a bias. Another bias could 
Table 1. Distribution of fishes in the Adyar Wetland complex during the survey (2007-2011)

\begin{tabular}{|c|c|c|c|c|c|c|c|c|c|}
\hline & & $\begin{array}{l}\text { Chem- } \\
\text { baram- } \\
\text { pakkam }\end{array}$ & $\begin{array}{l}\text { Upri- } \\
\text { ver }\end{array}$ & $\begin{array}{l}\text { Down- } \\
\text { river }\end{array}$ & Estuary & $\begin{array}{l}\text { Point of } \\
\text { conflu- } \\
\text { ence }\end{array}$ & $\begin{array}{l}\text { Srinva- } \\
\text { sapuram } \\
\text { Creek }\end{array}$ & $\begin{array}{c}\text { Adyar } \\
\text { Poonga } \\
\text { (east of } \\
\text { spillway) }\end{array}$ & $\begin{array}{c}\text { Adyar } \\
\text { Poonga } \\
\text { (west of } \\
\text { spillway) }\end{array}$ \\
\hline & Class: Chondrichthyes (Cartilaginous Fishes) & & & & & & & & \\
\hline & Order: Rajiformes (Rays) & & & & & & & & \\
\hline & Family: Rhinobatidae (Guitarfishes) & & & & & & & & \\
\hline \multirow[t]{2}{*}{1} & Grey Guitarfish Rhinobatus obtusus Muller \& Henle & - & - & - & + & + & - & - & - \\
\hline & Family: Dasyatidae (Stingrays) & & & & & & & & \\
\hline 2 & Honeycombed Stingray Dasyatis uarnak (Forsskal) & - & - & - & - & + & - & - & - \\
\hline \multirow[t]{4}{*}{3} & Taeniura sp. Muller \& Henle & - & - & - & - & + & - & - & - \\
\hline & Class: Osteichthyes (Bony Fishes) & & & & & & & & \\
\hline & Order: Osteoglossiformes & & & & & & & & \\
\hline & Family: Notopteridae (Featherbacks) & & & & & & & & \\
\hline \multirow[t]{3}{*}{4} & Grey Featherback Notopterus notopterus (Pallas) & + & - & - & - & - & - & - & - \\
\hline & Order: Clupeiformes (Herrings and allies) & & & & & & & & \\
\hline & Family: Clupeidae (Sardines and Shads) & & & & & & & & \\
\hline 5 & Kelee Shad Hilsa kelee (Cuvier) & - & - & - & + & - & - & - & - \\
\hline 6 & Bloch's Gizzard Shad Nematalosa nasus (Bloch) & - & - & - & + & + & + & - & - \\
\hline 7 & Goldstripe Sardinella Sardinella gibbosa (Bleeker) & - & - & - & + & - & - & - & - \\
\hline \multirow[t]{2}{*}{8} & White Sardine Escualosa thoracata (Valenciennes) & - & - & - & + & - & - & - & - \\
\hline & Family: Engraulidae (Anchovies) & & . & & & & & & \\
\hline 9 & Malabar Thryssa Thryssa malabarica (Bloch) & - & - & - & + & + & - & - & - \\
\hline \multirow[t]{3}{*}{10} & Moustached Thryssa Thryssa mystax (Schneider) & - & - & - & + & + & - & - & - \\
\hline & Order: Elopiformes & & & & & & & & \\
\hline & Family: Elopidae (Ladyfishes and Tenpounders) & & & & & & & & \\
\hline \multirow[t]{2}{*}{11} & Tenpounder Elops machnata (Forsskal) & - & - & - & + & + & + & - & - \\
\hline & Family: Albulidae (Bonefishes) & & & & & & & & \\
\hline \multirow[t]{2}{*}{12} & Bonefish Albula vulpes (Linnaeus) & - & - & - & + & + & - & - & - \\
\hline & Family: Megalopidae (Tarpons) & & & & & & & & \\
\hline \multirow[t]{3}{*}{13} & Indo-Pacific Tarpon Megalops cyprinoides (Broussonet) & - & - & - & + & - & + & + & + \\
\hline & Order: Anguilliformes (Eels) & & & & & & & & \\
\hline & Family: Anguillidae (Freshwater Eels) & & & & & & & & \\
\hline \multirow[t]{2}{*}{14} & Indian Shortfin Eel Anguilla bicolor McClelland & - & - & - & + & - & + & + & + \\
\hline & Family: Ophichthidae (Snake Eels) & & & & & & & & \\
\hline 15 & Ophichthus microcephalus (Day) & - & - & - & + & + & - & - & - \\
\hline \multirow[t]{3}{*}{16} & Rice-paddy Eel Pisodonophis boro (Hamilton) & - & - & - & + & - & - & - & - \\
\hline & Order: Gonorynchiformes & & & & & & & & \\
\hline & Family: Chanidae (Milk Fish) & & & & & & & & \\
\hline \multirow[t]{3}{*}{17} & Milk Fish Chanos chanos (Forsskal) & - & - & - & + & - & + & & \\
\hline & Order: Cypriniformes & & & & & & & & \\
\hline & Family: Cyprinidae (Carps and allies) & & & & & & & & \\
\hline 18 & Swamp Barb Puntius chola (Hamilton) & + & + & + & - & - & - & + & + \\
\hline 19 & Rosy Barb Puntius conchonius (Hamilton) & + & + & - & - & - & - & - & + \\
\hline 20 & Long-snouted Barb Puntius dorsalis (Jerdon) & + & + & - & - & - & - & - & - \\
\hline 21 & $\begin{array}{l}\text { Black-spot or Indian Tiger Barb Puntius filamentosus } \\
\text { (Valenciennes) }\end{array}$ & + & + & - & - & - & - & - & - \\
\hline
\end{tabular}




\begin{tabular}{|c|c|c|c|c|c|c|c|c|c|}
\hline & & $\begin{array}{l}\text { Chem- } \\
\text { baram- } \\
\text { pakkam }\end{array}$ & $\begin{array}{l}\text { Upri- } \\
\text { ver }\end{array}$ & $\begin{array}{l}\text { Down- } \\
\text { river }\end{array}$ & Estuary & $\begin{array}{l}\text { Point of } \\
\text { conflu- } \\
\text { ence }\end{array}$ & $\begin{array}{l}\text { Srinva- } \\
\text { sapuram } \\
\text { Creek }\end{array}$ & $\begin{array}{c}\text { Adyar } \\
\text { Poonga } \\
\text { (east of } \\
\text { spillway) }\end{array}$ & $\begin{array}{c}\text { Adyar } \\
\text { Poonga } \\
\text { (west of } \\
\text { spillway) }\end{array}$ \\
\hline 22 & Olive Barb Puntius sarana sarana (Hamilton-Buchanan) & + & - & - & - & - & - & - & - \\
\hline 23 & Spotfin or Swamp Barb Puntius sophore (Hamilton) & + & + & - & - & - & - & - & + \\
\hline 24 & Firefin or Two-spot Barb Puntius ticto (Hamilton) & + & - & - & - & - & - & - & - \\
\hline 25 & Kooli Barb Puntius vittatus Day & + & - & - & - & - & - & - & - \\
\hline 26 & Puntius mahecola (=P. amphibius) (Valenciennes) & + & - & - & - & - & - & - & - \\
\hline 27 & Puntius sharmai Menon \& Rema Devi & - & - & - & - & - & - & - & + \\
\hline 28 & Indian Glass Barb Laubuca laubuca (Hamilton) & + & - & - & - & - & - & - & - \\
\hline 29 & Bloch's Razorbelly Minnow Salmostoma clupeoides (Bloch) & + & - & - & - & - & - & - & - \\
\hline 30 & Indian Carplet Amblypharyngodon microlepis (Bleeker) & + & + & - & - & - & - & - & + \\
\hline 31 & Flying Barb Esomus danricus (Hamilton-Buchanan) & + & + & - & + & - & - & + & + \\
\hline 32 & Sri Lanka Flying Barb Esomus thermoicos (Valenciennes) & + & - & - & - & - & - & - & - \\
\hline 33 & Cauvery Rasbora Rasbora caverii (Jerdon) & + & - & - & - & - & - & - & - \\
\hline 34 & Blackline Rasbora Rasbora daniconius (Hamilton) & + & + & - & - & - & - & - & + \\
\hline 35 & Kalabans Labeo dero (Hamilton) & + & - & - & + & - & - & - & - \\
\hline \multirow[t]{2}{*}{36} & Catla Gibelion catla (Hamilton)** & + & + & - & + & - & - & - & - \\
\hline & Family: Cobitidae (Loaches) & & & & & & & & \\
\hline 37 & Guntea Loach Lepidocephalus guntea (Hamilton)*** & + & + & - & - & - & - & - & - \\
\hline \multirow[t]{3}{*}{38} & Malabar Loach Lepidocephalus thermalis (Valenciennes) & + & - & - & - & - & - & - & - \\
\hline & Order: Siluriformes (Catfishes) & & & & & & & & \\
\hline & Family: Bagridae & & & & & & & & \\
\hline 39 & Gangetic Mystus Mystus cavasius (Hamilton) & + & - & - & - & - & - & - & - \\
\hline 40 & Striped Dwarf Catfish Mystus vittatus (Bloch) & + & + & - & - & - & - & - & - \\
\hline 41 & Day's Mystus Mystus bleekeri (Day) & + & - & - & - & - & - & - & - \\
\hline \multirow[t]{2}{*}{42} & Mystus cf. gulio & + & + & + & + & - & + & + & + \\
\hline & Family: Siluridae & & & & & & & & \\
\hline \multirow[t]{2}{*}{43} & Shark Catfish Wallago attu (Schneider) & + & - & - & - & - & - & - & + \\
\hline & Family: Schilbeidae & & & & & & & & \\
\hline \multirow[t]{2}{*}{44} & Indian Potasi Neotropius atherinoides (Bloch) & + & - & - & - & - & - & - & - \\
\hline & Family: Heteropneustidae & & & & & & & & \\
\hline \multirow[t]{2}{*}{45} & Stinging Catfish Heteropneustes fossilis (Bloch) & + & + & - & - & - & - & - & + \\
\hline & Family: Ariidae & & & & & & & & \\
\hline 46 & Hamilton's Catfish Arius arius (Hamilton) & - & - & - & + & + & + & - & - \\
\hline 47 & Small-eyed Catfish Arius jella Day & - & - & - & + & + & - & - & - \\
\hline \multirow[t]{2}{*}{48} & Spotted Catfish Arius maculatus (Thunberg) & - & - & - & + & - & - & - & - \\
\hline & Family: Loricariidae (Suckermouth Armoured Catfish) & & & & & & & & \\
\hline 49 & Pterygoplichthys sp. ${ }^{*}$ & + & - & - & - & - & - & - & - \\
\hline \multirow[t]{3}{*}{50} & Pseudacanthicus sp.* & + & - & - & - & - & - & - & - \\
\hline & Order: Muguliformes & & & & & & & & \\
\hline & Family: Mugilidae (Mullets) & & & & & & & & \\
\hline 51 & Goldspot Mullet Liza parsia (Hamilton) & - & - & - & + & + & + & - & - \\
\hline 52 & Borneo Mullet Liza macrolepis (Smith) & - & - & - & + & + & + & + & - \\
\hline 53 & Tade Grey Mullet Liza tade (Forsskal) & - & - & - & + & + & - & - & - \\
\hline 54 & Flathead Grey Mullet Mugil cephalus Linnaeus & - & - & - & + & + & + & + & - \\
\hline
\end{tabular}




\begin{tabular}{|c|c|c|c|c|c|c|c|c|c|}
\hline & & $\begin{array}{l}\text { Chem- } \\
\text { baram- } \\
\text { pakkam }\end{array}$ & $\begin{array}{l}\text { Upri- } \\
\text { ver }\end{array}$ & $\begin{array}{l}\text { Down- } \\
\text { river }\end{array}$ & Estuary & $\begin{array}{c}\text { Point of } \\
\text { conflu- } \\
\text { ence }\end{array}$ & $\begin{array}{l}\text { Srinva- } \\
\text { sapuram } \\
\text { Creek }\end{array}$ & $\begin{array}{c}\text { Adyar } \\
\text { Poonga } \\
\text { (east of } \\
\text { spillway) }\end{array}$ & $\begin{array}{c}\text { Adyar } \\
\text { Poonga } \\
\text { (west of } \\
\text { spillway) }\end{array}$ \\
\hline & Order: Beloniformes & & & & & & & & \\
\hline & Family: Adrianichthyidae & & & & & & & & \\
\hline 55 & Ricefish Oryzias dancena (Hamilton) & + & - & - & - & - & - & - & - \\
\hline \multirow[t]{2}{*}{56} & Oryzias carnaticus (Jerdon) & + & - & - & - & - & - & - & - \\
\hline & Family: Belonidae (Fullbeak Garfishes) & & & & & & & & \\
\hline \multirow[t]{2}{*}{57} & Freshwater Garfish Xenentodon cancila (Hamilton) & + & + & - & - & - & - & - & - \\
\hline & Family: Hemiramphidae (Halfbeak garfishes) & & & & & & & & \\
\hline \multirow[t]{3}{*}{58} & $\begin{array}{l}\text { Congaturi Halfbeak Hyporhamphus limbatus } \\
\text { (Valenciennes) }\end{array}$ & + & - & - & - & - & - & - & - \\
\hline & Order: Cyprinodontiformes & & & & & & & & \\
\hline & Family: Aplocheilidae (Panchax Minnows) & & & & & & & & \\
\hline \multirow[t]{2}{*}{59} & Dwarf Panchax Aplocheilus parvus Raj***** & + & - & - & - & - & - & - & + \\
\hline & Family; Poeciliidae (Livebearers) & & & & & & & & \\
\hline 60 & Guppy Poecilia reticulata Peters* & - & - & - & - & - & - & - & + \\
\hline \multirow[t]{3}{*}{61} & Mosquitofish Gambusia affinis (Baird \& Girard)* & + & + & - & - & - & - & - & + \\
\hline & Order: Synbranchiformes & & & & & & & & \\
\hline & Family: Mastacembelidae (Spiny Eels) & & & & & & & & \\
\hline 62 & $\begin{array}{l}\text { Onestripe Spiny Eel Macrognathus aral (Bloch \& } \\
\text { Schneider) }\end{array}$ & + & - & - & - & - & - & - & - \\
\hline \multirow[t]{3}{*}{63} & Striped Spiny Eel Macrognathus pancalus Hamilton & + & - & - & - & - & - & - & - \\
\hline & Order: Perciformes & & & & & & & & \\
\hline & Family: Centropomidae (Sea Perch) & & & & & & & & \\
\hline \multirow[t]{2}{*}{64} & Giant Seaperch or Seabass Lates calcarifer (Bloch) & - & - & - & + & + & + & + & - \\
\hline & Family: Ambassidae (Glassfishes) & & & & & & & & \\
\hline 65 & $\begin{array}{l}\text { Commerson's Glassy Perchlet Ambassis commersoni } \\
\text { Cuvier }\end{array}$ & - & - & - & + & + & + & + & - \\
\hline 66 & Indian Glassy Fish Pseudambassis ranga (Hamilton) & + & - & - & - & - & - & - & - \\
\hline \multirow[t]{2}{*}{67} & Elongate Glass Fish Chanda nama (Hamilton) & + & - & - & - & - & - & - & - \\
\hline & Family: Sillaginidae (Sillagos) & & . & & & & & & \\
\hline \multirow[t]{2}{*}{68} & Silver Sillago Sillago sihama (Forsskal) & - & - & - & + & + & + & - & - \\
\hline & Family: Carangidae (Horse Mackerels) & & & & & & & & \\
\hline 69 & Tille Trevally Caranx tille Cuvier & - & - & - & + & + & - & - & - \\
\hline 70 & Yellowfin Jack Caranx ignobilis (Forsskal) & - & - & - & + & + & - & - & - \\
\hline 71 & Tille Jack Caranx sexfaciatus Quoy \& Gaimard & - & - & - & + & - & - & - & - \\
\hline 72 & Banded Scad Caranx para Cuvier & - & - & - & + & - & - & - & - \\
\hline \multirow[t]{2}{*}{73} & Alepes sp. & - & - & - & - & + & - & - & - \\
\hline & Family: Leiognathidae (Ponyfishes) & & & & & & & & \\
\hline 74 & Splendid Ponyfish Leiognathus splendens (Cuvier) & - & - & - & + & - & + & + & - \\
\hline 75 & Pugnose Ponyfish Secutor insidiator (Bloch) & - & - & - & + & - & + & - & - \\
\hline 76 & Deep Pugnose Ponyfish Secutor ruconius (Hamilton) & - & - & - & + & - & - & - & - \\
\hline \multirow[t]{2}{*}{77} & Toothed Ponyfish Gazza minuta (Bloch) & - & - & - & + & - & - & - & - \\
\hline & Family: Gerreidae (Mojarras) & & & & & & & & \\
\hline 78 & Whipfin Mojarra Gerres filamentosus Cuvier & - & - & - & + & - & - & - & - \\
\hline 79 & Common Mojarra Gerres oyena (Forsskal) & - & - & - & + & - & - & - & - \\
\hline 80 & Silvery Mojarra Gerres poeti Cuvier & - & - & - & + & - & - & - & - \\
\hline
\end{tabular}




\begin{tabular}{|c|c|c|c|c|c|c|c|c|c|}
\hline & & $\begin{array}{l}\text { Chem- } \\
\text { baram- } \\
\text { pakkam }\end{array}$ & $\begin{array}{l}\text { Upri- } \\
\text { ver }\end{array}$ & $\begin{array}{l}\text { Down- } \\
\text { river }\end{array}$ & Estuary & $\begin{array}{c}\text { Point of } \\
\text { conflu- } \\
\text { ence }\end{array}$ & $\begin{array}{l}\text { Srinva- } \\
\text { sapuram } \\
\text { Creek }\end{array}$ & $\begin{array}{l}\text { Adyar } \\
\text { Poonga } \\
\text { (east of } \\
\text { spillway) }\end{array}$ & $\begin{array}{c}\text { Adyar } \\
\text { Poonga } \\
\text { (west of } \\
\text { spillway) }\end{array}$ \\
\hline & Family: Pomadasyidae (Sweetlips) & & & & & & & & \\
\hline \multirow[t]{2}{*}{81} & Black Sweetlip Plectorhynchus nigrus (Cuvier) & - & - & - & - & + & - & - & - \\
\hline & Family: Polynemidae (Threadfins) & & & & & & & & \\
\hline \multirow[t]{2}{*}{82} & Common Threadfin Polydactulus plebius (Broussonet) & - & - & - & + & + & - & - & - \\
\hline & Family: Teraponidae (Perches and Grunters) & & & & & & & & \\
\hline 83 & Jarbua Terapon or Target Perch Terapon jarbua (Forsskal) & - & - & - & + & + & + & - & - \\
\hline \multirow[t]{2}{*}{84} & Small-scaled Banded Grunter Terapon puta Cuvier & - & - & - & + & + & - & - & - \\
\hline & Family: Mullidae (Goatfishes) & & & & & & & & \\
\hline \multirow[t]{2}{*}{85} & Yellow Goatfish Upeneus sulphureus Cuvier & - & - & - & - & + & - & - & - \\
\hline & Family: Ephippidae (Drepanes) & & & & & & & & \\
\hline \multirow[t]{2}{*}{86} & Banded Drepane Drepane longimana (Bloch \& Schneider) & - & - & - & - & + & - & - & - \\
\hline & Family: Scatophagidae (Butterfishes) & & & & & & & & \\
\hline \multirow[t]{2}{*}{87} & Spotted Scat Scatophagus argus (Linnaeus) & - & - & - & + & + & + & - & - \\
\hline & Family: Sphyraenidae (Barracudas) & & & & & & & & \\
\hline \multirow[t]{2}{*}{88} & Banded Barracuda Sphyraena jello Cuvier & - & - & - & + & + & - & - & - \\
\hline & Family: Siganidae (Rabbitfishes) & & & & & & & & \\
\hline \multirow[t]{2}{*}{89} & Streaky Spinefoot Siganus javus (Linnaeus) & - & - & - & + & + & + & - & - \\
\hline & Family: Cichlidae (Cichlids) & & & & & & & & \\
\hline 90 & Orange Chromide Etroplus maculatus (Bloch) & + & + & - & - & - & - & + & + \\
\hline \multirow[t]{2}{*}{91} & $\begin{array}{l}\text { Tilapia or Egyptian Mouthbreeder Oreochromis } \\
\text { mossambica* (Peters) }\end{array}$ & + & + & + & + & + & + & + & + \\
\hline & Family: Gobiidae (Gobies) & & & & & & & & \\
\hline \multirow[t]{2}{*}{92} & Tank Goby Glossogobius giurus (Hamilton) & + & - & - & - & - & - & - & + \\
\hline & Family: Belontiidae (Gouramies) & & & & & & & & \\
\hline 93 & Dwarf Gouramy Colisa lalia (Hamilton)*** & + & + & - & - & - & - & - & + \\
\hline 94 & Threespot Gouramy Trichogaster trichopterus (Pallas)* & + & + & - & - & - & - & - & + \\
\hline \multirow[t]{2}{*}{95} & Giant Gourami Osphronemus goramy Lacepede* & + & - & - & - & - & - & - & - \\
\hline & Family: Anabantidae (Climbing perch) & & & & & & & & \\
\hline \multirow[t]{2}{*}{96} & Climbing Perch Anabas testudineus (Bloch) & + & + & + & + & + & + & + & + \\
\hline & Family: Channidae (Snakeheads) & & & & & & & & \\
\hline 97 & Spotted Snakehead Channa punctatus (Bloch) & + & + & - & - & - & - & - & + \\
\hline \multirow[t]{2}{*}{98} & Striped Snakehead Channa striatus (Bloch) & + & + & - & - & - & - & - & + \\
\hline & Family: Stromateidae (Pomfrets) & & & & & & & & \\
\hline \multirow[t]{3}{*}{99} & Silver Pomfret Pampus argenteus (Euphrasen) & - & - & - & - & + & - & - & - \\
\hline & Order: Tetradontiformes & & & & & & & & \\
\hline & Family: Triacanthidae (Tripod Fishes) & & & & & & & & \\
\hline 100 & Tripod Fish Triacanthus brevirostris Schlegel & - & - & - & + & + & - & - & - \\
\hline 101 & Short-nosed Tripod Fish Triacanthus biaculeatus (Bloch) & - & - & - & + & + & - & - & - \\
\hline
\end{tabular}

$+=$ Present; - = Absent; ${ }^{*}=$ Exotics; ${ }^{* *}=$ Translocated species; $* * *=$ Species occuring due to the linking of waterways; ${ }^{* * *}=$ Species of controversial origin; $* * * * *=$ Reintroduction; $\quad=$ Freshwater; $\quad=$ Brackish and saline water 


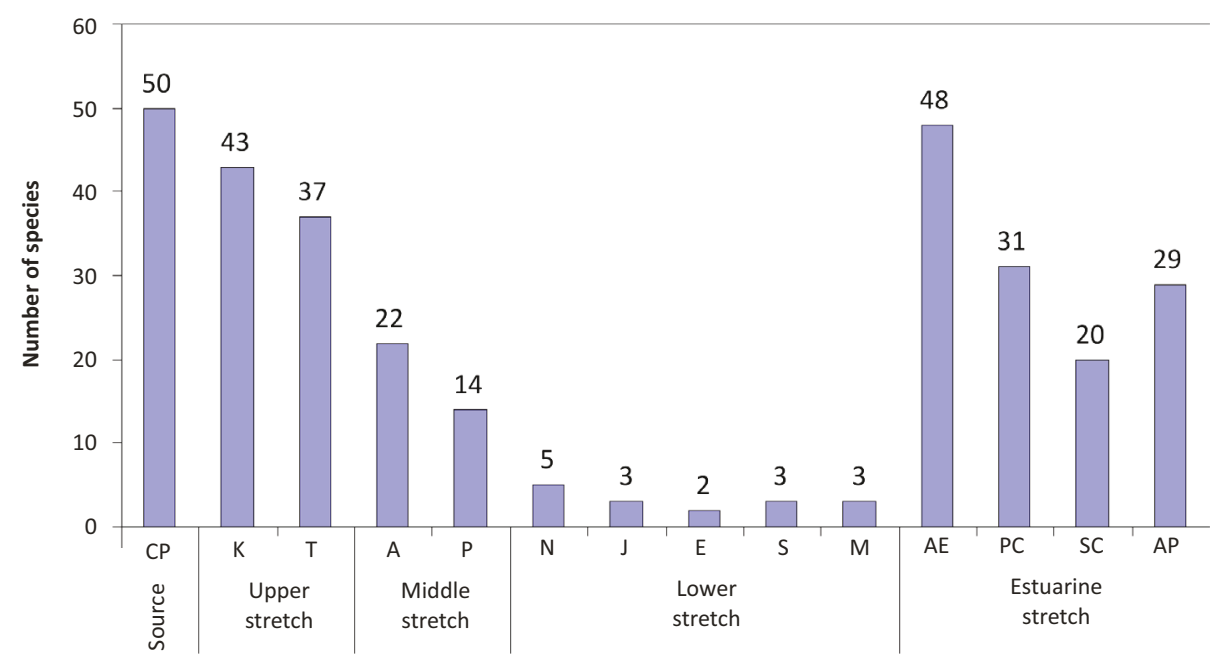

Figure 1. Distribution of fish in the Adyar wetland complex during the survey (2007-2011)

have arisen from fundamental sampling procedures - for example, fisheries oriented samplings may have focussed principally on commercially viable species. The present survey was the most comprehensive, intensive and longest running one-for a period of four years and three months-and hence may have recorded more diversity. Another eye opener is the Fishery Report from April 1949 to March 1950 (Anonymous 1950) which documented 73 species. What is pertinent is that 87 species recorded during earlier surveys were not encountered during this survey. For further details refer Table 2 and Fig. 2.

Twenty-three new records have been established for the estuarine reach. Among the findings was Caranx tille. Talwar \& Kacker (1984) mentioned that "there is no positive record of $C$. tille in our region (though mentioned in the literature)". Another notable finding was Mystus cf. gulio. The specimens encountered in the wetland showed a marked aberrant feature-viz., the maxillary barbels were very short. This is in stark contrast to the description by Jayaram $(2003,2006)$ who mentioned that the maxillary pair reached the middle or sometimes almost end of pelvic fins. This is ironic because the common name for Mystus gulio is the "Long Whiskered Catfish". Efforts are on to determine the taxonomic validity of the specimens. Nevertheless, morphometric values of the specimens have been presented here (Table 4). This is pertinent because Linder (2000) states "Mystus gulio: as presently understood, is a collection of brackish Mystus that likely represent many valid species" and the presence of a new taxon at the restoration site could have immeasurable conservation value.

The heartening factor is the colonization processes underway at the rehabilitation site. In 2007 only four species were recorded (Anonymous 2007) when the

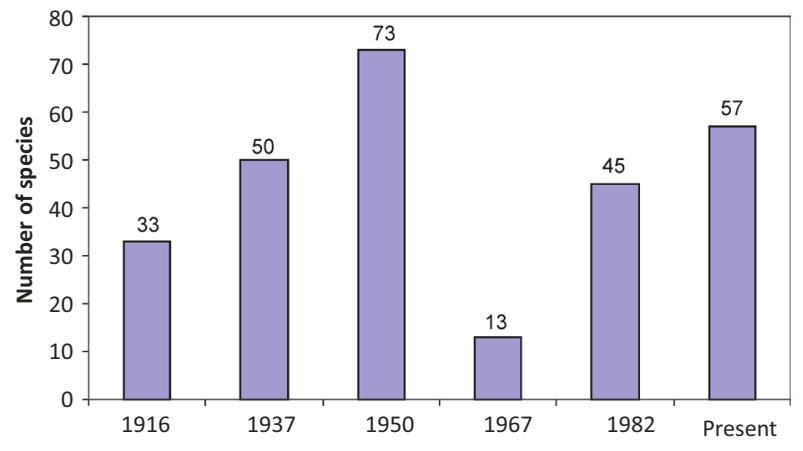

Figure 2. Total number species recorded in Adyar from 1916 to present.

site was an environmental disaster. After the removal of debris, prevention of pollution entering the site, removal of invasive Water Hyacinth Eichhornia crassipes, expanding the water spread and linking the water bodies, 13 species were recorded during the follow up survey from July 2007 to October 2008 (Ramanujam et al. 2008). Presently the count stands at 28. For further details refer Table 3 and Fig. 3.

The striking feature of the survey was the number of alien species encountered in the wetland. Two fish species listed as some of the "100 of the world's worst invasive alien species" (Lowe et al. 2000) occur in the wetland (and even the rehabilitation site)-viz., Mosquito Fish Gambusia affinis and Egyptian Mouthbreeder or Tilapia Oreochromis mossambica. Though it is unknown when the first was introduced [Ragunathan (1978) is the first record for the wetland], the introduction of $O$. mossambica and the aftereffects are well documented. Devadoss \& Chacko (1953) suggested the introduction of O. mossambica for fisheries purposes and 15,000 
Table 2. Fish known to occur in the Adyar estuarine reach: historical records in relation to the present survey

\begin{tabular}{|c|c|c|c|c|c|c|c|}
\hline & Species & 1916* & 1937 & 1950 & 1967 & 1982 & Present \\
\hline & Class: Chondrichthyes (Cartilaginous Fishes) & & & & & & \\
\hline & Order: Rajiformes (Rays) & & & & & & \\
\hline & Family: Rhinobatidae (Guitarfishes) & & & & & & \\
\hline \multirow[t]{2}{*}{1} & Grey Guitarfish Rhinobatus obtusus Muller \& Henle & - & - & - & - & - & + \\
\hline & Family: Dasyatidae (Stingrays) & & & & & & \\
\hline 2 & Honeycombed Stingray Dasyatis uarnak (Forsskal) & - & - & - & - & - & + \\
\hline \multirow[t]{4}{*}{3} & Taeniura sp. Muller \& Henle & - & - & - & - & - & + \\
\hline & Class: Osteichthyes (Bony Fishes) & & & & & & \\
\hline & Order: Clupeiformes (Herrings and allies) & & & & & & \\
\hline & Family: Clupeidae (Sardines and Shads) & & & & & & \\
\hline 4 & Kelee Shad Hilsa kelee (Cuvier) & - & - & - & - & - & + \\
\hline 5 & Bloch's Gizzard Shad Nematalosa nasus (Bloch) & - & - & - & - & - & + \\
\hline 6 & Goldstripe Sardinella Sardinella gibbosa (Bleeker) & - & + & + & - & - & + \\
\hline 7 & Fringescale Sardinella Sardinella fimbriata (Valenciennes) & - & - & - & - & + & - \\
\hline 8 & White Sardinella Sardinella albella (Valenciennes) & - & - & - & - & + & - \\
\hline \multirow[t]{2}{*}{9} & White Sardine Escualosa thoracata (Valenciennes) & - & - & - & - & - & + \\
\hline & Family: Chirocentridae & & & & & & \\
\hline \multirow[t]{2}{*}{10} & Wolf Herring Chirocentrus dorab (Forsskal) & - & - & - & - & + & - \\
\hline & Family: Engraulidae (Anchovies) & & & & & & \\
\hline 11 & Malabar Thryssa Thryssa malabarica (Bloch) & - & - & - & - & + & + \\
\hline 12 & Moustached Thryssa Thryssa mystax (Hamilton) & - & - & - & - & + & + \\
\hline 13 & Gangetic Anchovy Thryssa purava (Hamilton) & + & + & + & + & + & - \\
\hline 14 & Commerson's Anchovy Stolephorus commersonii Lacepede & - & + & + & $?$ & - & - \\
\hline \multirow[t]{3}{*}{15} & Indian Anchovy Stolephorus indicus (van Hasselt) & - & - & - & ? & + & - \\
\hline & Order: Elopiformes & & & & & & \\
\hline & Family: Elopidae (Ladyfishes and Tenpounders) & & & & & & \\
\hline \multirow[t]{2}{*}{16} & Tenpounder Elops machnata (Forsskal) & + & + & + & + & + & + \\
\hline & Family: Albulidae (Bonefishes) & & & & & & \\
\hline \multirow[t]{2}{*}{17} & Bonefish Albula vulpes (Linnaeus) & - & - & - & - & - & + \\
\hline & Family: Megalopidae (Tarpons) & & & & & & \\
\hline \multirow[t]{3}{*}{18} & Indo-Pacific Tarpon Megalops cyprinoides (Broussonet) & + & + & + & + & + & + \\
\hline & Order: Anguilliformes (Eels) & & & & & & \\
\hline & Family: Anguillidae (Freshwater Eels) & & & & & & \\
\hline \multirow[t]{2}{*}{19} & Indian Shortfin Eel Anguilla bicolor McClelland & + & - & - & - & + & + \\
\hline & Family: Ophichthidae (Snake Eels) & & & & & & \\
\hline 20 & Ophichthus microcephalus (Day) & - & - & + & - & - & + \\
\hline \multirow[t]{3}{*}{21} & Rice-paddy Eel Pisodonophis boro (Hamilton) & - & - & - & - & - & + \\
\hline & Order: Gonorynchiformes & & & & & & \\
\hline & Family: Chanidae (Milk Fish) & & & & & & \\
\hline \multirow[t]{3}{*}{22} & Milk Fish Chanos chanos (Forsskal) & - & + & + & + & + & + \\
\hline & Order: Cypriniformes & & & & & & \\
\hline & Family: Cyprinidae (Carps and allies) & & & & & & \\
\hline 23 & Long-snouted Barb Puntius dorsalis (Jerdon) & + & + & + & ? & - & - \\
\hline 24 & $\begin{array}{l}\text { Black-spot or Indian Tiger Barb Puntius filamentous } \\
\text { (Valenciennes) }\end{array}$ & + & - & + & $?$ & - & - \\
\hline 25 & Spotfin or Swamp Barb Puntius sophore (Hamilton) & + & + & + & $?$ & - & - \\
\hline 26 & Firefin or Two-spot Barb Puntius ticto (Hamilton) & - & - & + & ? & - & - \\
\hline 27 & Kooli Barb Puntius vittatus Day & + & - & - & $?$ & + & - \\
\hline
\end{tabular}




\begin{tabular}{|c|c|c|c|c|c|c|c|}
\hline & Species & 1916* & 1937 & 1950 & 1967 & 1982 & Present \\
\hline 28 & Puntius mahecola (=P. amphibius) (Valenciennes) & + & + & + & ? & - & - \\
\hline 29 & Bloch's Razorbelly Minnow Salmostoma clupeoides (Bloch) & + & - & + & - & - & - \\
\hline 30 & Silver Razorbelly Minnow Salmostoma acinaces (Valenciennes) & - & - & + & - & - & - \\
\hline 31 & Indian Carplet Amblypharyngodon microlepis (Bleeker) & + & - & + & - & - & - \\
\hline 32 & Flying Barb Esomus danricus (Hamilton) & + & - & + & - & - & - \\
\hline 33 & Kalabans Labeo dero (Hamilton) & - & - & - & - & - & + \\
\hline 34 & Fringe-lipped Peninsular Carp Labeo fimbriatus (Bloch) & - & - & + & - & - & - \\
\hline 35 & Malabar Labeo Labeo dussumieri (Valenciennes) & - & - & - & - & + & - \\
\hline 36 & Reba Carp Cirrhinus reba (Hamilton) & + & - & + & - & - & - \\
\hline 37 & Catla Gibelion catla (Hamilton) & - & - & - & - & - & + \\
\hline \multirow[t]{3}{*}{38} & Hamilton's Baril Barilius bendelisis (Hamilton) & - & - & + & - & - & - \\
\hline & Order: Siluriformes (Catfishes) & & & & & & \\
\hline & Family: Bagridae & & & & & & \\
\hline 39 & Keletius Mystus Mystus keletius (Valenciennes) & + & + & + & - & - & - \\
\hline 40 & Striped Dwarf Catfish Mystus vittatus (Bloch) & + & + & + & + & - & - \\
\hline \multirow[t]{2}{*}{41} & Mystus cf gulio** & - & + & + & + & - & + \\
\hline & Family: Clariidae (Airbreathing Catfish) & & & & & & \\
\hline \multirow[t]{2}{*}{42} & Magur Clarius batrachus (Linnaeus) & + & - & - & - & - & - \\
\hline & Family: Plotosidae (Eeltail Catfish) & & & & & & \\
\hline 43 & Canine Catfish Eel Plotosus canius Hamilton & - & + & + & - & - & - \\
\hline \multirow[t]{2}{*}{44} & Striped Eel Catfish Plotosus lineatus (Thunberg) & - & - & - & - & + & - \\
\hline & Family: Siluridae & & & & & & \\
\hline \multirow[t]{2}{*}{45} & Shark Catfish Wallago attu (Schneider) & - & - & + & - & - & - \\
\hline & Family: Ariidae & & & & & & \\
\hline 46 & Hamilton's Catfish Arius arius (Hamilton) & - & - & - & ? & - & + \\
\hline 47 & Small-eyed Catfish Arius jella Day & - & - & - & ? & - & + \\
\hline 48 & Spotted Catfish Arius maculates (Thunberg) & + & - & - & ? & + & + \\
\hline \multirow[t]{3}{*}{49} & Dussumier's Catfish Arius dussumieri Valenciennes & - & - & - & ? & + & - \\
\hline & Order: Muguliformes & & & & & & \\
\hline & Family: Mugilidae (Mullets) & & & & & & \\
\hline 50 & Goldspot Mullet Liza parsia (Hamilton) & - & - & + & ? & + & + \\
\hline 51 & Borneo Mullet Liza macrolepis (Smith) & - & - & - & $?$ & + & + \\
\hline 52 & Tade Grey Mullet Liza tade (Forsskal) & - & - & - & ? & + & + \\
\hline 53 & Diamondscale Grey Mullet Liza vaigiensis (Quoy \& Gaimard) & - & - & - & ? & + & - \\
\hline 54 & Bluespot Grey Mullet Valamugil seheli (Forsskal) & - & - & - & ? & + & - \\
\hline \multirow[t]{3}{*}{55} & Flathead Grey Mullet Mugil cephalus Linnaeus & - & + & + & $?$ & + & + \\
\hline & Order: Beloniformes & & & & & & \\
\hline & Family: Adrianichthyidae (Ricefishes) & & & & & & \\
\hline \multirow[t]{2}{*}{56} & Ricefish Oryzias dancena (Hamilton)** & - & + & + & - & - & - \\
\hline & Family: Belonidae (Fullbeak Garfishes) & & & & & & \\
\hline \multirow[t]{2}{*}{57} & Freshwater Garfish Xenentodon cancila (Hamilton) & + & - & + & - & - & + \\
\hline & Family: Hemiramphidae (Halfbeak garfishes) & & & & & & \\
\hline \multirow[t]{3}{*}{58} & Congaturi Halfbeak Hyporhamphus limbatus (Valenciennes) & - & - & + & - & - & - \\
\hline & Order: Cyprinodontiformes & & & & & & \\
\hline & Family: Aplocheilidae (Panchax Minnows) & & & & & & \\
\hline \multirow[t]{3}{*}{59} & Dwarf Panchax Aplocheilus parvus Raj** & - & + & + & - & - & - \\
\hline & Order: Synbranchiformes & & & & & & \\
\hline & Family: Mastacembelidae (Spiny Eels) & & & & & & \\
\hline
\end{tabular}




\begin{tabular}{|c|c|c|c|c|c|c|c|}
\hline & Species & 1916* & 1937 & 1950 & 1967 & 1982 & Present \\
\hline 60 & Onestripe Spiny Eel Macrognathus aral (Bloch \& Schneider) & - & - & + & + & - & - \\
\hline \multirow[t]{3}{*}{61} & Striped Spiny Eel Macrognathus pancalus Hamilton & + & - & + & - & - & - \\
\hline & Order: Scorpaeniformes & & & & & & \\
\hline & Family: Platycephalidae (Flatheads) & & & & & & \\
\hline 62 & Indian Flathead Platycephalus indicus (Linnaeus) & - & + & + & - & - & - \\
\hline 63 & Large-spined Flathead Platycephalus rodericensis Cuvier & - & - & - & - & + & - \\
\hline 64 & Rough Flathead Platycephalus scaber (Linnaeus) & - & + & - & - & - & - \\
\hline \multirow[t]{3}{*}{65} & Serrated Flathead Platycephalus serratus Cuvier & - & - & - & - & + & - \\
\hline & Order: Perciformes & & & & & & \\
\hline & Family: Centropomidae (Sea Perch) & & & & & & \\
\hline \multirow[t]{2}{*}{66} & Giant Seaperch or Seabass Lates calcarifer (Bloch) & + & - & + & + & - & + \\
\hline & Family: Ambassidae (Glassfishes) & & & & & & \\
\hline 67 & Commerson's Glassy Perchlet Ambassis commersoni Cuvier & & + & + & ? & + & + \\
\hline 68 & Myops Glassy Perchlet Ambassis miops Gunther & + & + & + & $?$ & - & - \\
\hline 69 & Indian Glassy Fish Pseudambassis ranga (Hamilton) & + & - & + & - & - & - \\
\hline \multirow[t]{2}{*}{70} & Elongate Glass Fish Chanda nama (Hamilton) & - & - & + & - & - & - \\
\hline & Family: Apogonidae & & & & & & \\
\hline 71 & Twobelt Cardinal Apogon taeniatus (Cuvier) & - & - & + & - & - & - \\
\hline \multirow[t]{2}{*}{72} & Ring-tailed Cardinal Fish Apogon aureus Lacepede & - & - & + & - & - & - \\
\hline & Family: Serranidae (Groupers) & & & & & & \\
\hline 73 & Greasy Grouper Epinephelus tauvina (Forsskal) & - & - & - & ? & + & - \\
\hline 74 & Malabar Reefcod Epinephelus malabaricus (Schneider) & - & - & - & ? & + & - \\
\hline \multirow[t]{2}{*}{75} & Red-banded Grouper Epinephelus fasciatus (Forsskal) & - & + & + & ? & - & - \\
\hline & Family: Sillaginidae (Sillagos) & & & & & & \\
\hline \multirow[t]{2}{*}{76} & Silver Sillago Sillago sihama (Forsskal) & - & + & + & + & - & + \\
\hline & Family: Carangidae (Horse Mackerels) & & & & & & \\
\hline 77 & Tille Trevally Caranx tille Cuvier & - & - & - & - & - & + \\
\hline 78 & Yellowfin Jack Caranx ignoblis (Forsskal) & - & - & - & - & - & + \\
\hline 79 & Tille Jack Caranx sexfaciatus Quoy \& Galmard & - & - & - & - & - & + \\
\hline 80 & Banded Scad Caranx para Cuvier & - & - & - & - & - & + \\
\hline 81 & Malabar Trevally Carangoides malabaricus (Bloch) & - & - & - & - & + & - \\
\hline 82 & Alepes sp. & - & - & - & - & - & + \\
\hline \multirow[t]{2}{*}{83} & Threadfin Trevally Alectis ciliaris (Bloch) & - & + & + & - & - & - \\
\hline & Family: Lutjanidae (Snappers) & & & & & & \\
\hline 84 & John's Snapper Lutjanus johni (Bloch) & - & + & + & $?$ & + & - \\
\hline \multirow[t]{2}{*}{85} & Bigeye Snapper Lutjanus lineolatus (Ruppell) & - & - & + & $?$ & - & - \\
\hline & Family: Leiognathidae (Ponyfishes) & & & & & & \\
\hline 86 & Splendid Ponyfish Leiognathus splendens (Cuvier) & - & - & - & - & - & + \\
\hline 87 & Orangefin Ponyfish Leiognathus bindus (Valenciennes) & - & - & - & - & + & - \\
\hline 88 & Striped Ponyfish Leiognathus fasciatus (Lacepede) & - & - & - & - & + & - \\
\hline 89 & Pugnose ponyfish Secutor insidiator (Bloch) & - & - & + & - & - & + \\
\hline 90 & Deep Pugnose Ponyfish Secutor ruconius (Hamilton) & - & - & - & - & - & + \\
\hline \multirow[t]{2}{*}{91} & Toothed Ponyfish Gazza minuta (Bloch) & - & - & - & - & - & + \\
\hline & Family: Gerreidae (Mojarras) & & & & & & \\
\hline 92 & Whipfin Mojarra Gerres filamentosus Cuvier & - & - & - & + & - & + \\
\hline 93 & Common Mojarra Gerres oyena (Forsskal) & - & - & + & - & - & + \\
\hline \multirow[t]{2}{*}{94} & Silvery Mojarra Gerres poeti Cuvier & - & - & - & - & - & + \\
\hline & Family: Lethrinidae (Breams) & & & & & & \\
\hline
\end{tabular}




\begin{tabular}{|c|c|c|c|c|c|c|c|}
\hline & Species & 1916* & 1937 & 1950 & 1967 & 1982 & Present \\
\hline \multirow[t]{2}{*}{95} & Starry Pigface Bream Lethrinus nebulosa (Forsskal) & - & - & - & - & + & - \\
\hline & Family: Pomadasyidae (Sweetlips) & & & & & & \\
\hline \multirow[t]{2}{*}{96} & Black Sweetlip Plectorhynchus nigrus (Cuvier) & - & - & - & - & - & + \\
\hline & Family: Polynemidae (Threadfins) & & & & & & \\
\hline 97 & Common Threadfin Polydactulus plebius (Broussonet) & - & - & - & - & - & + \\
\hline 98 & Sevenfinger Threadfin Polydactylus heptadactylus (Cuvier) & - & - & - & - & + & - \\
\hline 99 & Indian Threadfin Polydactylus indicus (Shaw) & - & - & - & - & + & - \\
\hline \multirow[t]{2}{*}{100} & Fourfinger Threadfin Eleutheronema tetradactylum (Shaw) & - & + & + & - & - & - \\
\hline & Family: Teraponidae (Perches and Grunters) & & & & & & \\
\hline 101 & Jarbua Terapon or Target Perch Terapon jarbua (Forsskal) & - & + & + & + & + & + \\
\hline 102 & Small-scaled Banded Grunter Terapon puta Cuvier & - & + & + & - & + & + \\
\hline \multirow[t]{2}{*}{103} & Four-lined Terapon Pelates quadrilineatus (Bloch) & - & + & - & - & - & - \\
\hline & Family: Mullidae (Goatfishes) & & & & & & \\
\hline \multirow[t]{2}{*}{104} & Yellow Goatfish Upeneus sulphureus Cuvier & - & + & + & - & - & + \\
\hline & Family: Ephippidae (Drepanes) & & & & & & \\
\hline \multirow[t]{2}{*}{105} & Banded Drepane Drepane longimana (Bloch \& Schneider) & - & - & - & - & - & + \\
\hline & Family: Scatophagidae (Butterfishes) & & & & & & \\
\hline \multirow[t]{2}{*}{106} & Spotted Scat Scatophagus argus (Linnaeus) & - & + & + & - & - & + \\
\hline & Family: Sphyraenidae (Barracudas) & & & & & & \\
\hline 107 & Banded Barracuda Sphyraena jello Cuvier & - & + & - & - & - & + \\
\hline 108 & Great Barracuda Sphyraena barracuda (Walbaum) & - & - & - & - & + & - \\
\hline \multirow[t]{2}{*}{109} & William's Barracuda Sphyraena bleekeri Williams & - & - & - & - & + & - \\
\hline & Family: Siganidae (Rabbitfishes) & & & & & & \\
\hline 110 & Streaky Spinefoot Siganus javus (Linnaeus) & - & - & + & - & + & + \\
\hline \multirow[t]{2}{*}{111} & White-spotted Spinefoot Siganus canaliculatus (Park) & - & - & - & - & + & - \\
\hline & Family: Cichlidae (Cichlids) & & & & & & \\
\hline 112 & Orange Chromide Etroplus maculatus (Bloch) & + & + & + & - & - & + \\
\hline 113 & Banded Pearlspot Etroplus suratensis (Bloch) & + & + & + & + & - & - \\
\hline \multirow[t]{2}{*}{114} & $\begin{array}{l}\text { Tilapia or Egyptian Mouthbreeder Oreochromis mossambica } \\
\text { (Peters) }\end{array}$ & - & - & - & + & + & + \\
\hline & Family: Gobiidae (Gobies) & & & & & & \\
\hline 115 & Tank Goby Glossogobius giurus (Hamilton) & + & + & + & - & - & - \\
\hline 116 & Tail-eyed Goby Parachaeturichthys polynema (Bleeker) & - & + & + & - & - & - \\
\hline 117 & Horny Goby Yongeichthys criniger (Valenciennes) & - & + & + & - & - & - \\
\hline 118 & Mangrove Goby Psammogobius biocellatus (Valenciennes) & - & - & - & - & + & - \\
\hline 119 & Hasselt's Goby Callogobius haseltti (Bleeker) & - & - & - & - & + & - \\
\hline 120 & Sharptail Goby Oligolepis acutipennis (Valenciennes) & + & + & - & - & - & - \\
\hline 121 & Meggit's Goby Bathygobius meggitti (Hora \& Mukerji) & - & + & - & - & - & - \\
\hline 122 & Indo-Pacific Tropical Sand Goby Favonigobius reichei (Bleeker) & + & + & + & - & - & - \\
\hline 123 & Spotted Green Goby Acentrogobius viridipunctatus (Valenciennes) & - & + & - & - & - & - \\
\hline 124 & Oxyurichthys tentacularis (Valenciennes) & + & + & + & - & - & - \\
\hline 125 & Apocryptichthys sp. & - & + & - & - & - & - \\
\hline 126 & Scartelos cantoris (Day) & - & - & + & - & - & - \\
\hline 127 & Boddart's Google-eyed Goby Boleophthalmus boddaerti (Pallas) & - & + & + & - & - & - \\
\hline 128 & Dusky Sleeper Eleotris fusca (Forster) & + & + & + & - & - & - \\
\hline 129 & Atlantic Mudskipper Periophthalmus barbarus (Linnaeus) & - & + & + & - & - & - \\
\hline \multirow[t]{2}{*}{130} & Pearse's Mudskipper Periophthalmus novemradiatus (Hamilton) & - & + & + & - & - & - \\
\hline & Family: Blennidae & & & & & & \\
\hline 131 & Zebra Blenny Omobranchus zebra (Bleeker) & - & + & - & - & - & - \\
\hline
\end{tabular}




\begin{tabular}{|c|c|c|c|c|c|c|c|}
\hline & Species & $1916 *$ & 1937 & 1950 & 1967 & 1982 & Present \\
\hline & Family: Anabantidae (Climbing perch) & & & & & & \\
\hline \multirow[t]{2}{*}{132} & Climbing Perch Anabas testudineus (Bloch) & + & - & + & - & - & + \\
\hline & Family: Channidae (Snakeheads) & & & & & & \\
\hline 133 & Spotted Snakehead Channa punctatus (Bloch) & + & - & + & ? & - & - \\
\hline 134 & Striped Snakehead Channa striatus (Bloch) & + & - & + & ? & - & - \\
\hline \multirow[t]{2}{*}{135} & Asiatic Snakehead Channa gachua (Hamilton) & + & - & - & ? & - & - \\
\hline & Family: Stromateidae (Pomfrets) & & & & & & \\
\hline \multirow[t]{3}{*}{136} & Silver Pomfret Pampus argenteus (Euphrasen) & - & - & - & - & - & + \\
\hline & Order: Tetradontiformes & & & & & & \\
\hline & Family: Triacanthidae (Tripod Fishes) & & & & & & \\
\hline 137 & Tripod Fish Triacanthus brevirostris Schlegel & - & + & + & - & - & + \\
\hline \multirow[t]{2}{*}{138} & Short-nosed Tripod Fish Triacanthus biaculeatus (Bloch) & - & - & - & - & - & + \\
\hline & Family: Tetraodontidae (Puffers) & & & & & & \\
\hline 139 & Milkspotted Puffer Chelonodon patoca (Hamilton) & - & + & + & - & - & - \\
\hline \multirow[t]{2}{*}{140} & Smooth Blaasop Lagocephalus inermis (Temminck \& Schlegel) & - & + & + & - & - & - \\
\hline & Family; Soleidae (Tongue Soles) & & & & & & \\
\hline 141 & $\begin{array}{l}\text { Large-toothed Flounder Pseudorhombus arsius (Hamilton - } \\
\text { Buchanan) }\end{array}$ & - & - & + & - & - & - \\
\hline 142 & Day's Tongue Sole Cynoglossus dispar Day & - & - & + & - & - & - \\
\hline 143 & Gangetic Tongue Sole Cynoglossus cynoglossus (Hamilton) & - & - & - & - & + & - \\
\hline 144 & Long Tongue Sole Cynoglossus lingua Hamilton & - & - & - & - & + & - \\
\hline
\end{tabular}

+ = Present; - = Absent; ? = Only genus or group mentioned; species are conjenctural (hence the symbol '?')

* - Raj (1916) mentions "....... my examination being confined to the rivers Cooum and Adyar....". Hence it is conjenctured that species listed as "estuarine", "occurring within tidal influence", "brackishwater" and "backwater" were encountered by him in both the Cooum and Adyar estuaries

** - Present systematics have been applied and nomenclature standardised [for example, Aplocheilus parvus was considered a synonym of $A$. blochii but Eapen (2007) has conclusively proved that species are distinct and $A$. parvus occurs along the east coast whereas $A$. blochii is restricted to the western region. The same applies to Oryzias spp. which have been revised recently (Roberts 1998)] The identity of the tentatively named Mystus cf. gulio needs to be confirmed based on revisionary work

specimens were introduced below Saidapet Road Bridge in December 1957 (Evangeline 1967b). As early as 1958 it was reported from fishermen's catches in the estuary (Chacko \& Venketaswami 1958) and in 1964-1965 it had overtaken the mullet fishery - the mullet catch accounted for $19.02 \%$, whereas the tilapia catch was 25.17\%, an increase of over 5\% (Evangeline 1967b). During the present survey the related Etroplus suratensis was not encountered and neither was it mentioned in an earlier report (Nammalwar 1982). Yet records state that it "was one of the most economically important fish of the Adyar river" (Anonymous 1950). The situation is alarming because two closely related alien species have been reported, viz.: Oreochromus aureus from Pallikaranai marsh in Chennai (Knight \& Devi 2009) and O. niloticus in Chembarampakkam (Knight 2010b). In addition, the highly aggressive hybrid 'Flowerhorn' Cichlasomax Amphilophus has been encountered in Chennai waterways (Knight 2010a), and if it gets established, is sure to spread to other wetlands, not only within Chennai, but even the Godavary and Krishna rivers due to linkages. Other exotics encountered were the suckermouth armoured catfishes Pterygoplichthys sp. and Pseudacanthicus sp., Guppy Poecilia reticulata, Threespot Gouramy Trichogaster trichopterus and Giant Gouramy Osphronemus goramy. Poecilia reticulata was recorded during the survey period July 2007-October 2008 at the rehabilitation site but not after that. It is interesting that Gambusia affinis was not recorded during that time and it is possible that it displaced $P$. reticulata at a later date.

While the problem of exotics is addressed from time to time in literature, little is said of fish from other parts of India, especially the large number of north Indian carps (introduced to boost inland fisheries) that have invaded areas where they do not occur naturally (Daniels 2006) and Gibelion catla is a typical example of such a translocated species. The linking of rivers has led to some species like Lepidocephalus guntea colonizing new areas. This species, a native of the Godavary and Krishna rivers (David 1963; Jayaram 1995), was encountered in sizable quantities during the July 2007-October 2008 survey phase at Chembarampakkam. At one time it was believed that $L$. guntea had replaced the native $L$. thermalis (Ramanujam et al. 2008) since the latter were not encountered at that 
Table 3. Fish of Adyar Poonga recorded during the three phases of this survey

\begin{tabular}{|c|c|c|c|c|c|}
\hline & Species & $\begin{array}{l}\text { Mar- } \\
\text { June } \\
2007\end{array}$ & $\begin{array}{c}\text { July } \\
2007- \\
\text { Oct } \\
2008\end{array}$ & $\begin{array}{c}\text { Nov } \\
2008- \\
\text { June } \\
2010\end{array}$ & $\begin{array}{c}\text { Jul } \\
2010- \\
\text { June } \\
2011\end{array}$ \\
\hline 1 & Megalops cyrpinoides & + & + & + & + \\
\hline 2 & Anguilla bicolor & - & + & + & + \\
\hline 3 & Chanos chanos & - & - & + & + \\
\hline 4 & Puntius chola & - & - & + & + \\
\hline 5 & Puntius conchonius & - & - & + & + \\
\hline 6 & Puntius sophore & - & - & + & + \\
\hline 7 & Puntius sharmai & - & - & + & + \\
\hline 8 & $\begin{array}{l}\text { Amblypharyngodon } \\
\text { microlepis }\end{array}$ & - & - & + & + \\
\hline 9 & Esomus danricus & - & - & + & + \\
\hline 10 & Rasbora daniconius & - & - & + & + \\
\hline 11 & Mystus cf. gulio & + & + & + & + \\
\hline 12 & Wallago attu & - & - & - & + \\
\hline 13 & Heteropneustes fossilis & - & + & + & + \\
\hline 14 & Liza microlepis & - & - & + & + \\
\hline 15 & Mugil cephalus & - & + & + & + \\
\hline 16 & Aplocheilus parvus* & - & - & + & + \\
\hline 17 & Poecilia reticulata** & - & + & - & - \\
\hline 18 & Gambusia affinis** & - & - & + & + \\
\hline 19 & Lates calcarifer & - & - & + & + \\
\hline 20 & Ambassis commersoni & - & + & + & + \\
\hline 21 & Leiognathus splendens & - & - & + & + \\
\hline 22 & Etroplus maculatus & - & - & + & + \\
\hline 23 & $\begin{array}{l}\text { Oreochromis } \\
\text { mossambica** }\end{array}$ & + & + & + & + \\
\hline 24 & Glossogobius giurus & - & - & + & + \\
\hline 25 & Colisa Ialia & - & + & + & + \\
\hline 26 & $\begin{array}{l}\text { Trichogaster } \\
\text { trichopterus** }\end{array}$ & - & + & + & + \\
\hline 27 & Anabas testudineus & + & + & + & + \\
\hline 28 & Channa punctatus & - & + & + & + \\
\hline 29 & Channa striatus & - & + & + & + \\
\hline
\end{tabular}

$+=$ Present; - = Absent; ${ }^{*}$ - Introduced in July 2009; ${ }^{* *}$ - Exotics

time and even earlier (Daniels \& Rajagopal 2004), but subsequently many specimens of $L$. thermalis were found during the later phase of surveys. Though pollution is presently touted to be the greatest threat to the wetland, it might be that homogenisation and colonization by alien species can be the greater threat to biodiversity. The fact is that invasive alien species are the second major cause of extinction of native and endemic species (Wilcove et al. 1998) and in the long run, if the wetland is restored to a semblance of its former self (and there are instances of

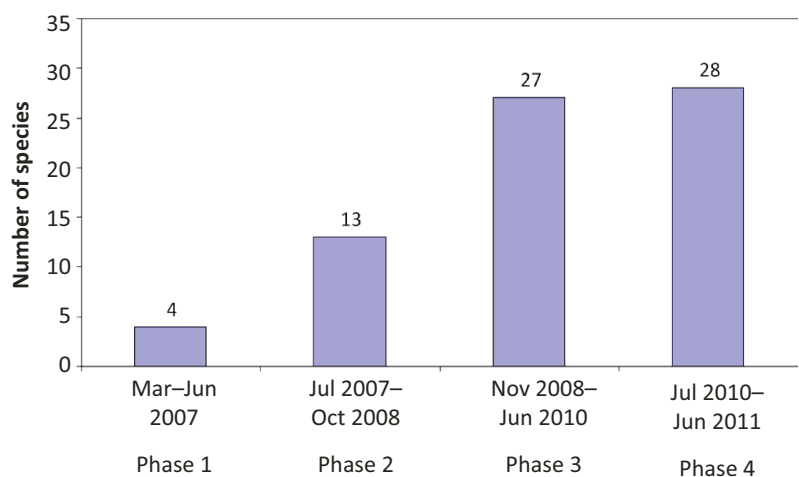

Figure 3. Fish diversity increase at the rehabilitation site during the four phases of the survey

the Thames, Sienne and other polluted waterways being restored since the technology and precedents exist), it will be the hardy aliens that will colonise and dominate newly restored habitats. Since there have been too few successful precedents of removal of alien fish, their removal may not only be expensive but unsuccessful since methodologies to cope with the phenomenon are still in their infancy on a global scale.

The occurrence of the Dwarf Gouramy Colisa lalia is controversial with some authorities claiming that it is not native to this part of India [Raghunathan (1978) had recorded Colisa fasciata at Chembarampakkam] and others favouring the opinion that it is indigenous to the region and populations boosted by the aquarium trade releases (R.J.R. Daniels pers. comm. 2010). The Dwarf Panchax Aplocheilus parvus, a native of the fresh waters of the east coast, was introduced as a larvicidal species into an artificial pond in June 2009 and has since spread into the wetland.

\section{REFERENCES}

Anonymous (1950). Rural Piscicultural Scheme. Progress Report (Period: $1^{\text {st }}$ April 1949 to $31^{\text {st }}$ March 1950). Indian Council of Agricultural Research, Part 6: 64pp.

Anonymous (2007). Adyar Poonga - Draft Ecological Restoration Plan. Submitted by Adyar Poonga Trust. Report prepared by the lead consultants, Pitchandikulam Forest Consultants to the Adyar Poonga Trust, Part 1. 208pp.

Bai, M.M., K.R. Devi \& T.J. Indra (2007). Fish as biological indicator in Cooum River. Indian Hydrobiology 10: 263-267.

Blasco, F. \& P. Legris (1972). Dry Evergreen Forest of Point Calimere and Marakanam. Journal of the Bombay Natural History Society 70 : 279-294.

Castro, P. \& M.E. Huber (1997). Marine Biology - $2^{\text {nd }}$ Edition. WCB/ McGraw-Hill Companies Inc., 450pp.

Chacko, P.I. \& A.D. Venkataswamy (1958). Development of brackishwater fisheries in Madras State. Ind - Com Journal 12: 157-159.

Daniels, R.J.R. (2002). Freshwater Fishes of Peninsular India. Universities Press (India) Private Limited, Hyderabad, 288pp+75figs.

Daniels, R.J.R. (2006). Introduced fishes: a potential threat to the 
Table 4. Morphometric values for Mystus cf. gulio encountered in Adyar estuary and creek [n=5].

\begin{tabular}{|c|c|c|c|c|c|c|c|}
\hline & Values & Specimen 1 & Specimen 2 & Specimen 3 & Specimen 4 & Specimen 5 & Mean \pm SD \\
\hline 1 & Total length & 198 & 190 & 162 & 175 & 150 & $175 \pm 19.67$ \\
\hline 2 & Standard length & 169 & 155 & 125 & 145 & 117 & $142 \pm 21.34$ \\
\hline 3 & Head length & 46.8 & 41.1 & 34.8 & 33.8 & 31.7 & $37.64 \pm 6.20$ \\
\hline 4 & Head depth & 23.6 & 21.7 & 20.9 & 21.7 & 18.1 & $21.2 \pm 2.00$ \\
\hline 5 & Body depth & 43.9 & 39.5 & 35.4 & 33.9 & 25.9 & $35.72 \pm 6.73$ \\
\hline 6 & Predorsal hypural distance & 64.2 & 58.9 & 47.7 & 53.7 & 47.2 & $54.34 \pm 7.31$ \\
\hline 7 & Dorsal hypural distance & 104.9 & 95.4 & 77.1 & 90.6 & 70.6 & $87.72 \pm 13.86$ \\
\hline 8 & Caudal peduncle length & 27.2 & 26.7 & 23.1 & 26.5 & 21.8 & $25.06 \pm 2.44$ \\
\hline 9 & Caudal peduncle depth & 24.9 & 22.1 & 18.3 & 18.9 & 18.3 & $20.5 \pm 2.92$ \\
\hline 10 & Length of maxillary barbel & 89.0 & 85.0 & 60.0 & 81.0 & 65.0 & $76 \pm 12.77$ \\
\hline 11 & Eye diameter & 8.6 & 6.7 & 6.4 & 6.5 & 5.7 & $6.78 \pm 1.08$ \\
\hline 12 & Inter-orbital distance & 19.2 & 16.0 & 12.3 & 14.1 & 12.8 & $14.88 \pm 2.81$ \\
\hline 13 & Inter-narial distance & 10.6 & 8.9 & 7.5 & 6.9 & 5.7 & $7.92 \pm 1.89$ \\
\hline
\end{tabular}

native freshwater fishes of peninsular India. Journal of the Bombay Natural History Society 103: 346-348.

Daniels, R.J.R. \& B. Rajagopal (2004). Fishes of Chembarampakkam Lake - a wetland in the outskirts of Chennai. Zoos' Print Journal 19(5): 1481-1483; http://dx.doi.org/10.11609/JoTT.ZPJ.1041.1481-3

David, A. (1963). Studies on fish and fisheries in the Godavary and Krishna River systems - Part 1. Proceedings of the National Academy of Sciences, India 33: 263-283.

Devadoss, D.D.P. \& P.I. Chacko (1953). Introduction of the exotic cichlid fish, Tilapia mossambica Peters, in Madras. Current Science 22: 29.

Eapen, A. (2007). Systematics and larvivorous potential of Indian fishes of the genus Aplocheilus McClelland (Pisces: Cypridontiformes) with special reference to Aplocheilus parvus Raj. PhD Thesis, University of Madras, 207pp.

Evangeline, G. (1967a). Chanos culture at the Brackishwater Fish Farm, Adyar. Madras Journal of Fisheries 3: 68-115.

Evangeline, G. (1967b). Trend in the fisheries of the Adyar Estuary from April 1963 to March 1965. Madras Journal of Fisheries 4: 1-20.

Jayaram, K.C. (1981). The Freshwater Fishes of India, Pakistan, Bangladesh, Burma and Sri Lanka - a Handbook. Zoological Survey of India, $475 \mathrm{pp}+13 \mathrm{pls}$.

Jayaram, K.C. (1995). The Krishna River System: A Bioresources Survey. Records of the Zoological Survey of India, 167pp+9pls.

Jayaram, K.C. (2006). Catfishes of India. Narendra Publishing House, Delhi, 383pp+11pls.

Jayaram, K.C. (2010). The Freshwater Fishes of the Indian Region - $2^{\text {nd }}$ Edition. Narendra Publishing House, Delhi, 616pp+39pls.

Knight, J.D.M. (2010a). Invasive ornamental fish: a potential threat to aquatic biodiversity in peninsular India. Journal of Threatened Taxa 2(2): 700-704; http://dx.doi.org/10.11609/JoTT.02179.700-4

Knight, J.D.M. (2010b). Addressing the Wallacean Shortfall: an updated checklist of ichthyofauna of Chembarampakkam Tank. Taprobanica 2: 25-29.

Knight, J.D.M. \& K.R. Devi (2009). Record of Oreochromis aureus (Steindachner, 1864) (Teleostei: Perciformes: Cichlidae) in the natural waters of Tamil Nadu. Taprobanica 1: 126-129.

Linder, R.S. (2000). The Catfishes of Asia. <www. catfishstudygroup.org/ pdf/catfishes_of_asia> 4pp.

Lowe, S., M. Browne \& S. Boudjelas (2000). 100 of the world's worst invasive alien species. IUCN / SSC Invasive Species Specialist Group, Auckland, New Zealand, 11pp.

Meher-Homji, V.M. (1974-1975). The natural history of Pondicherry and its environs. Revue Historique de Pondicherry 12: 45-50.
Nammalwar, P. (1982). Some aspects of ecophysiology and biological concern of the Estuarine Mullet Liza microlepis (Smith) with reference to heavy metals pollution. PhD Thesis, University of Madras, Department of Zoology, 216pp.

Narayanan, K. (1980). Hydrobiological study of the River Cooum in Madras, S. India with special reference to Aquaculture. PhD Thesis, University of Madras, Madras, 196pp.

Panikkar, N.K. \& R.G. Aiyar (1937). The brackish-water fauna of Madras. Proceedings of the Indian Academy of Sciences - Section B 6(5): 284337; http://dx.doi.org/10.1007/BF03051463

Raghunathan, M.B. (1978). Studies on seasonal tanks in Tamil Nadu. 1: Chembarampakkam Tank. The Indian Journal of Zootony 19: 81-85.

Raj, B.S. (1916). Notes on the freshwater fish of Madras. Records of the Indian Museum 12: 249-294.

Ramanujam, M.E., K.R. Devi, T.J. Indra \& T. Murugavel (2008). Vertebrate diversity survey of the Adyar wetland complex (20072008). Report submitted by Pitchandikulam Forest Consultants to Adyar Poonga Trust, 79pp.

Ramanujam, M.E., K.R. Devi, T.J. Indra \& T. Murugavel (2010). Vertebrate survey at Adyar creek and estuary. Report submitted by Pitchandikulam Forest Consultants to Chennai Rivers Restoration Trust 67pp+16pls.

Roberts, T.R. (1998). Systematic observations on tropical Asian medakas or ricefishes of the genus Oryzias, with descriptions of four new species. Ichthyological Research 45(3): 213-224; http://dx.doi. org/10.1007/BF02673919

Selvam, V., V. Hariprasad, R. Mohan \& R. Ramasubramaniam (1994). Diurnal variations in the water quality of sewage polluted Adayar mangrove water, east coast of India. Indian Journal of Marine Sciences 23: 94-97.

Sivakumari, K., K. Jayamalini, V. Kalaiarasi \& M. Sultana (2005). Variations in hydrographic factors of Adyar Estuary during different seasons. Nature Environment and Pollution Technology (formerly Journal of Environment and Pollution) 4: 212-221.

Talwar, P.K. \& R.K. Kacker (1984). Commercial Sea Fishes of India. Zoological Survey of India, Calcutta, 997pp.

Talwar, P.K. \& A.G. Jhingran (1991). Inland Fishes of India and Adjacent Countries - Volume 1 \& 2. Oxford and IBH Publishing Co. Pvt. Ltd., 1158pp.

Wilcove, D.S., D. Rothstein, J. Dubow, A. Phillips \& E. Losos (1998). Quantifying threats to imperiled species in the United States. BioScience 48(8): 607-615. 


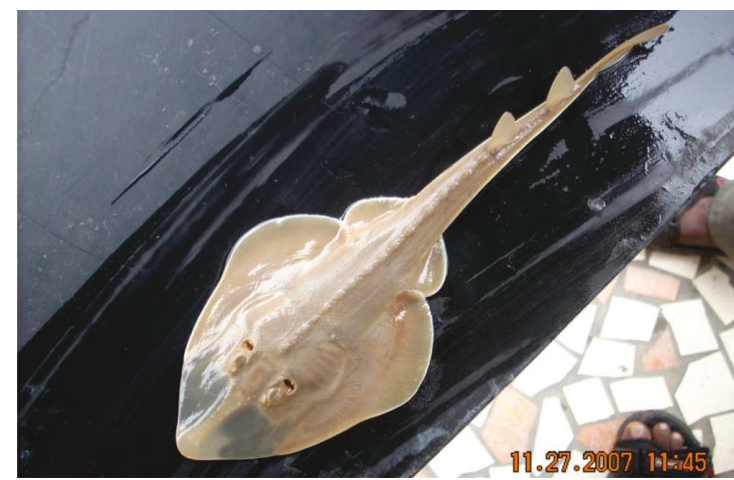

Image 1. Grey Guitarfish Himantura obtusus

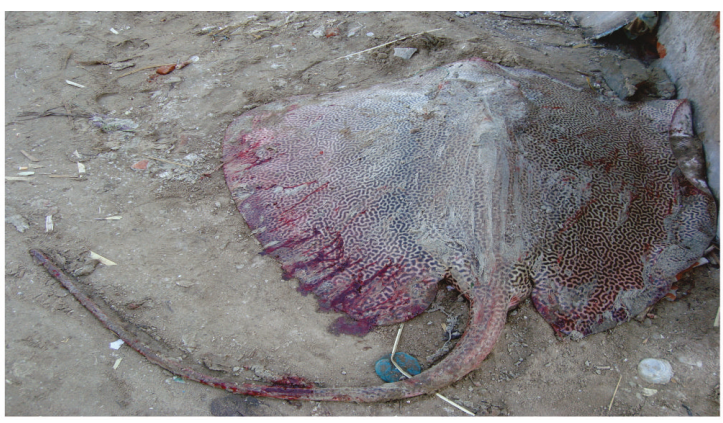

Image 3. Honeycombed Stingray Himantura uarnak

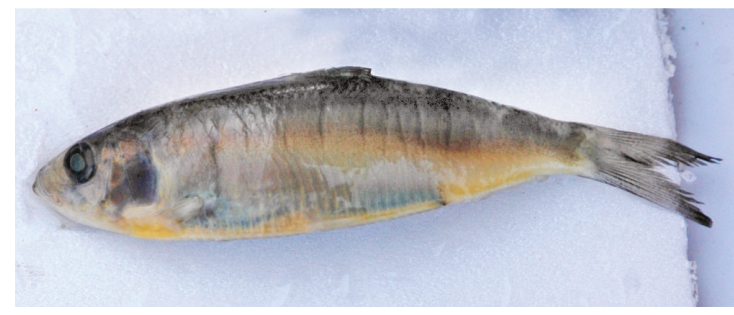

Image 5. Kelee Shad Hilsa kelee

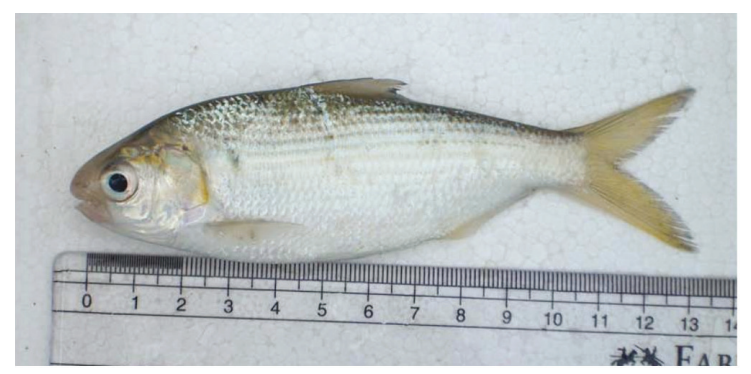

Image 7. Bloch's Gizzard Shad Nematalosa nasus

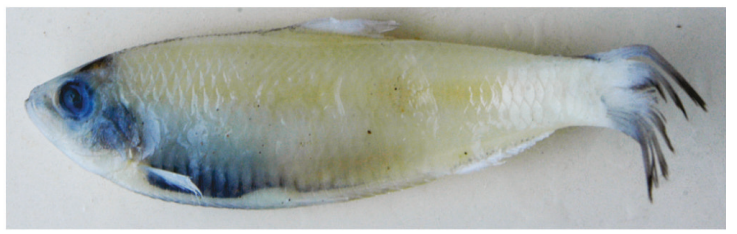

Image 9. White Sardine Escualosa thoracata

Photo credits for all images: (c) A. Lakshmikanthan

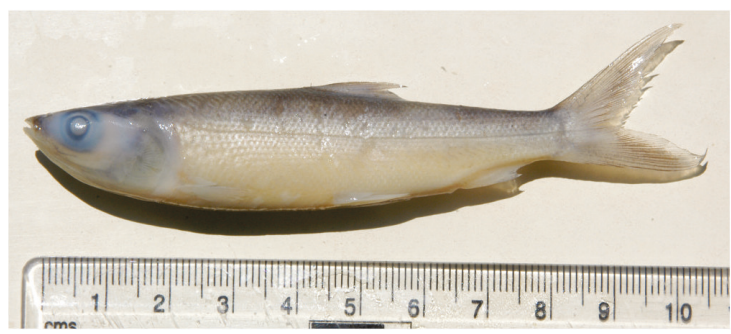

Image 2. Bonefish Albula vulpes

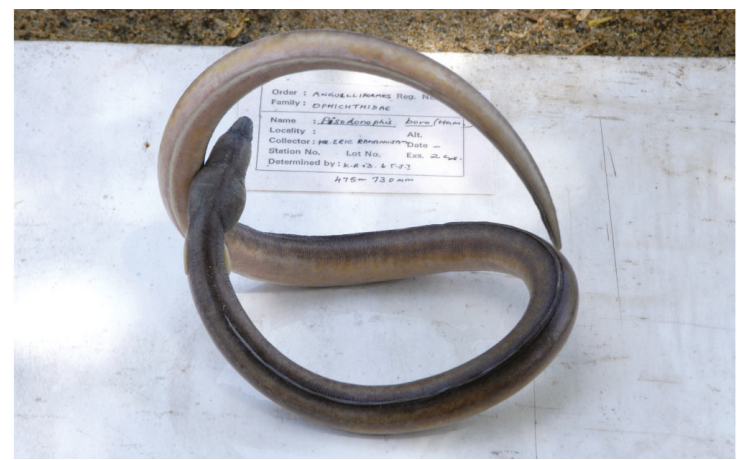

Image 4. Rice-paddy Eel Pisodonophis boro

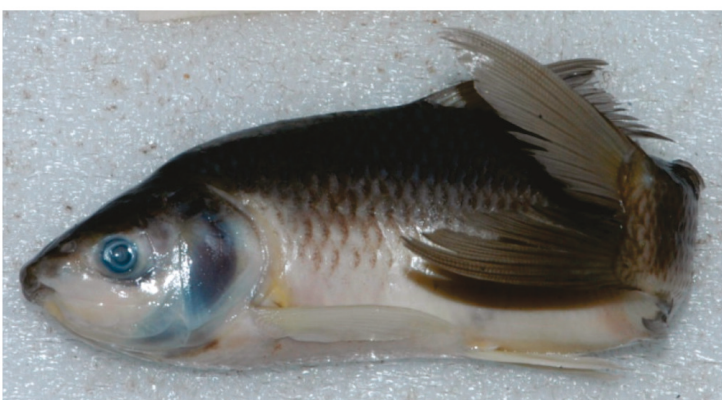

Image 6. Kalabans Bangana dero

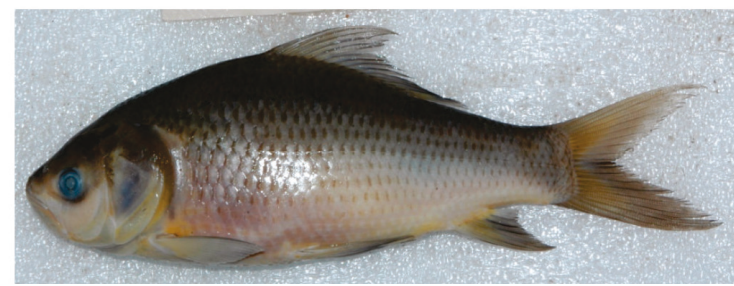

Image 8. Catla Gibelion catla

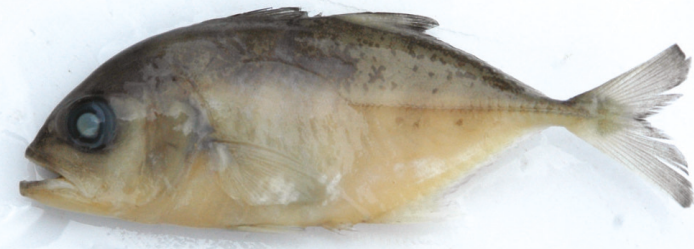

Image 10- Tile Trevally Caranx tille 


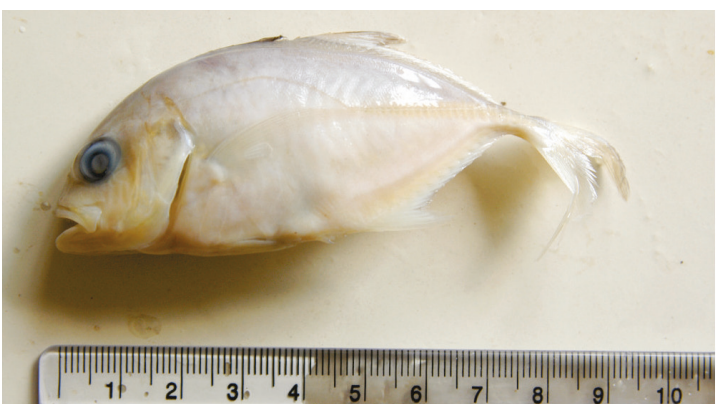

Image 11. Yellowfin Jack Caranx ignoblis

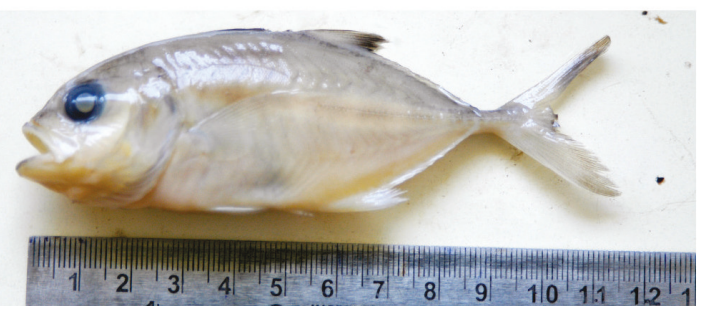

Image 13. Tille Jack Caranx sexfaciatus

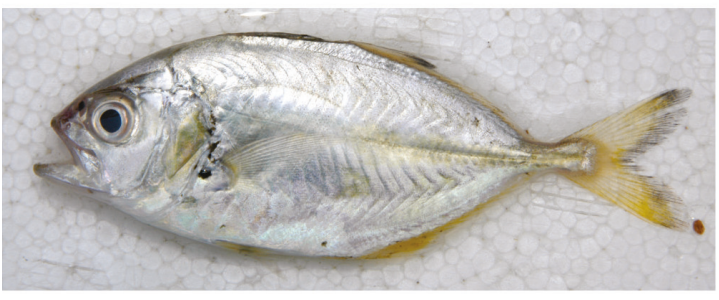

Image 15. Banded Scad Alepes kleinii

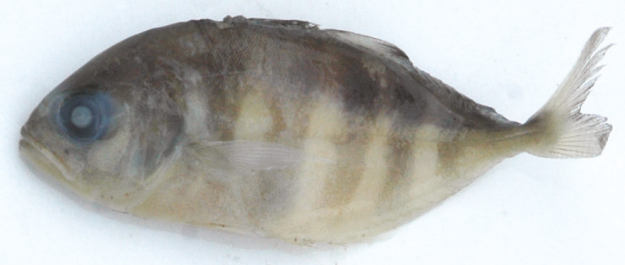

Image 17. Whitemouth Jack uraspis helvola

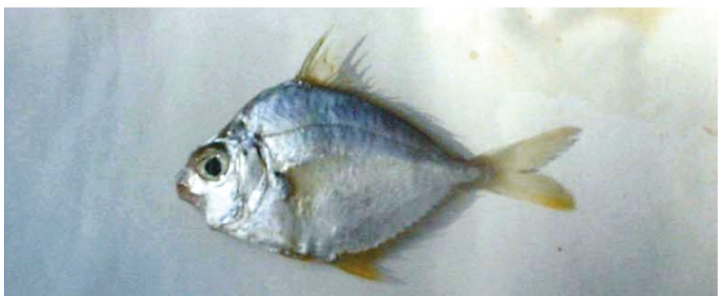

Image 19. Splendid Ponyfish Leiognathus splendens

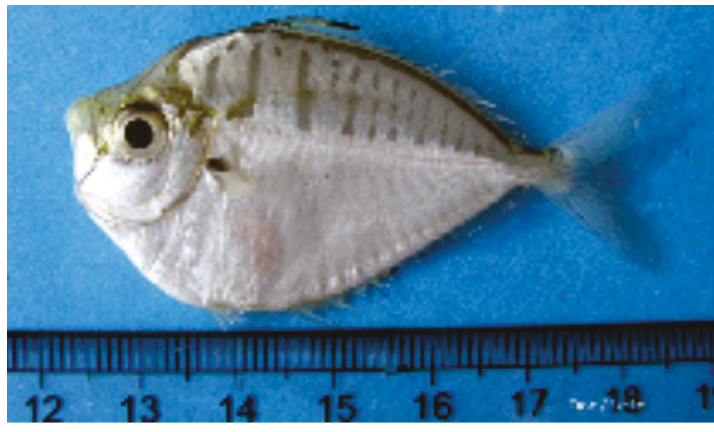

Image 12. Deep Pugnose Ponyfish Secutor ruconius

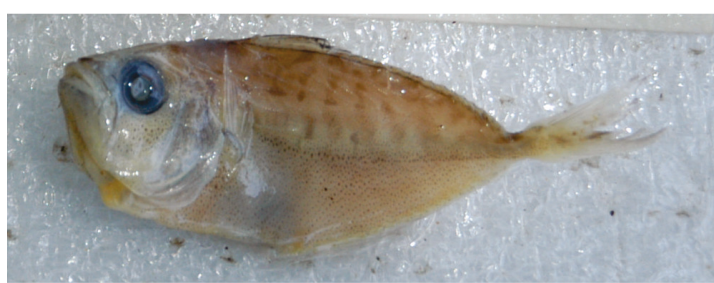

Image14. Toothed Ponyfish Gazza minuta

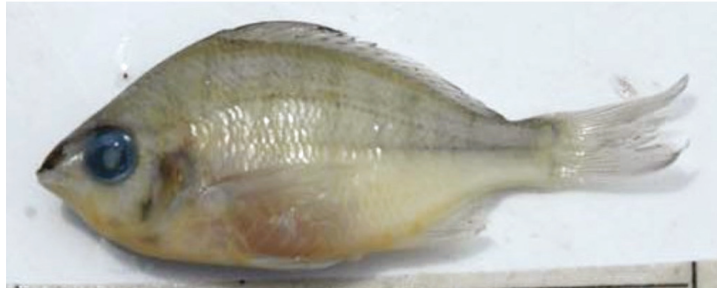

Image 16. Silvery Mojarra Gerres longirostris

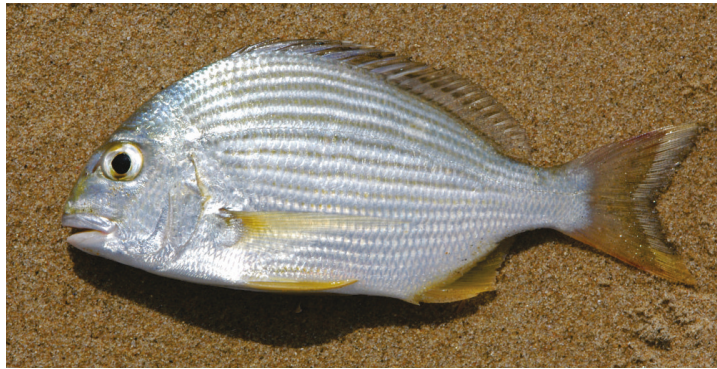

Image 18. Black Sweetlip Plectorhynchus gibbosus

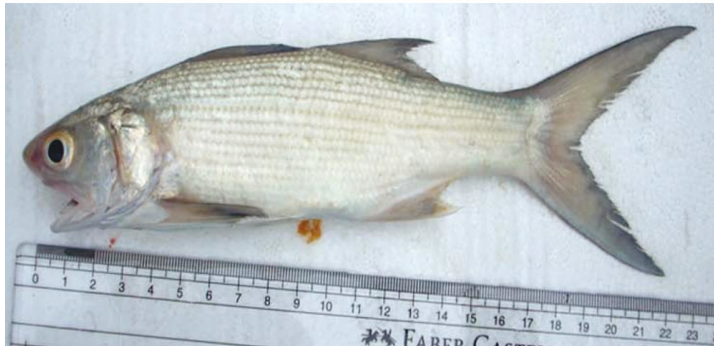

Image 20. Common Threadfin Polydactulus plebius 


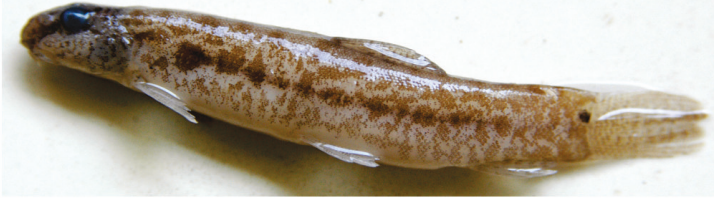

Image 21. Guntea Loach Lepidocephalus guntea

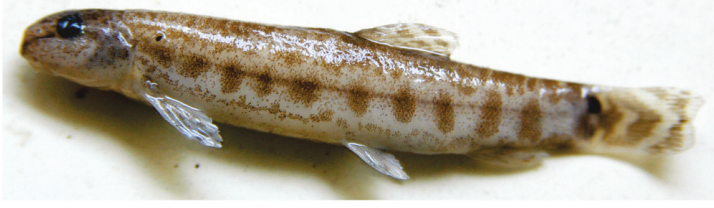

Image 23. Malabar Loach Lepidocephalus thermalis

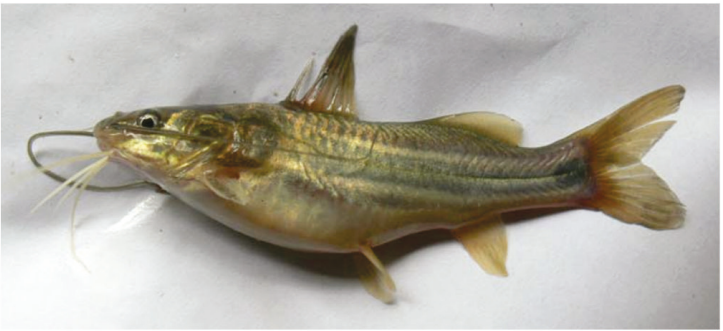

Image 25. Striped Dwarf Catfish Mystus vittatus

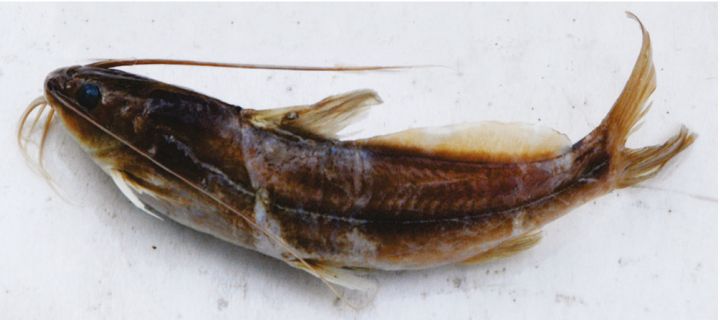

Image 27. Day's Mystus Mystus bleekeri

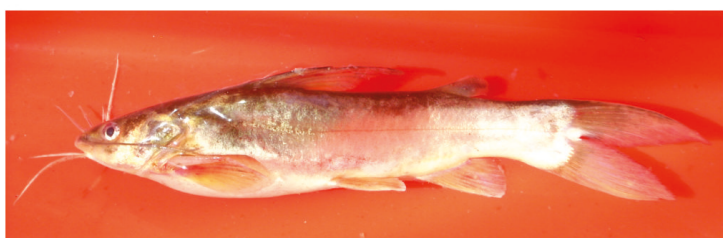

Image 29. Mystus cf. gulio

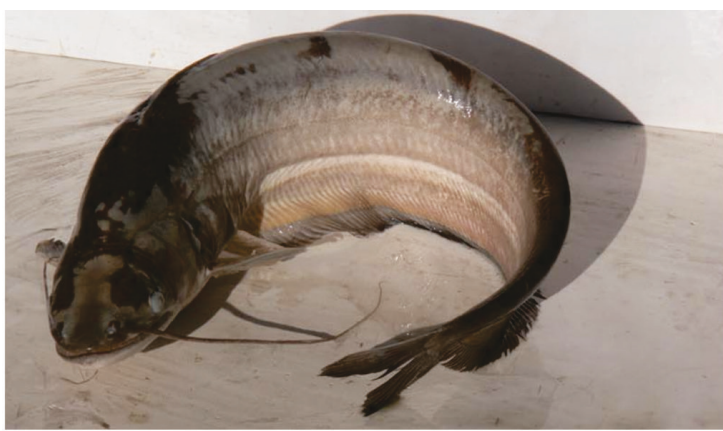

Image 31. Shark Catfish Wallago attu

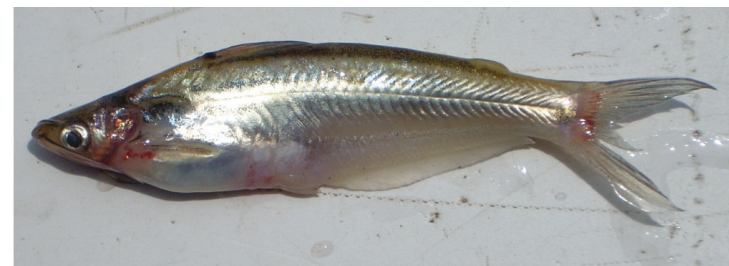

Image 22. Indian Potasi Neotropius atherinoides

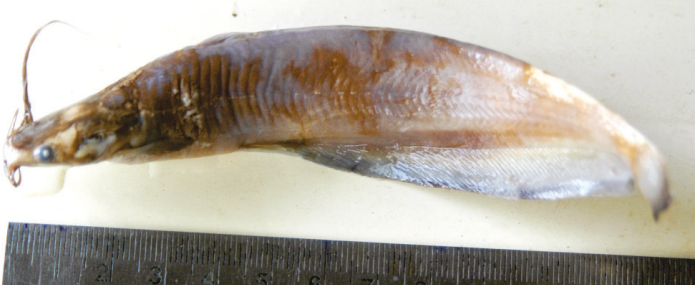

Image 24. Stinging Catfish Heteropneustes fossilis

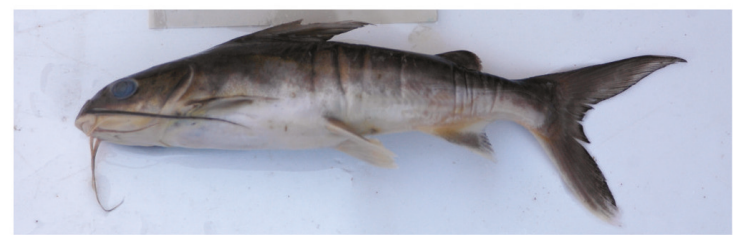

Image 26. Hamilton's Catfish Arius arius

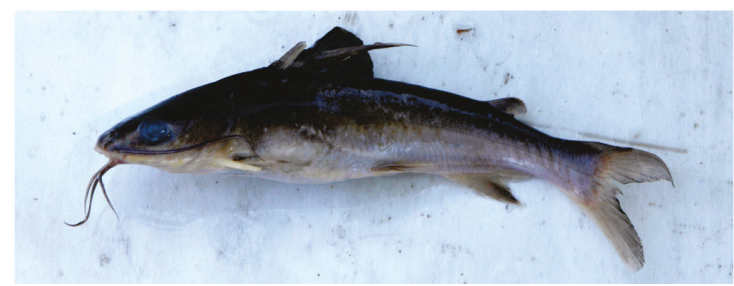

Image 28. Small-eyed Catfish Arius jella

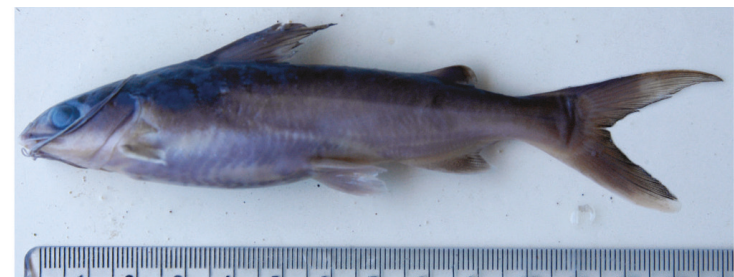
Image 30. Spotted Catfish Arius maculates

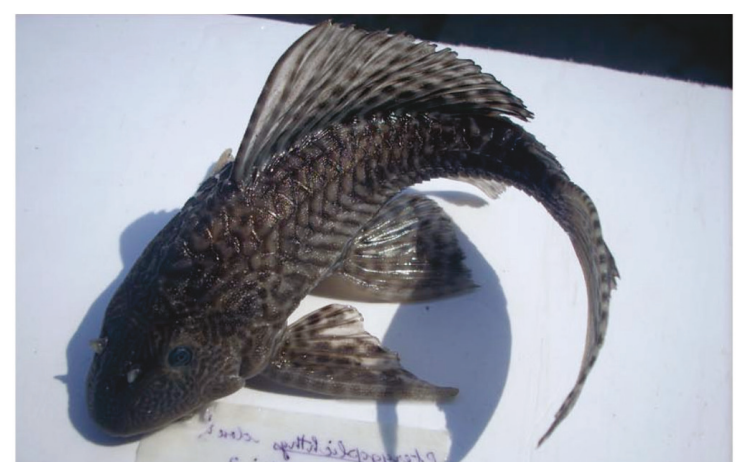

Image 32. Pterygoplichthys sp. 


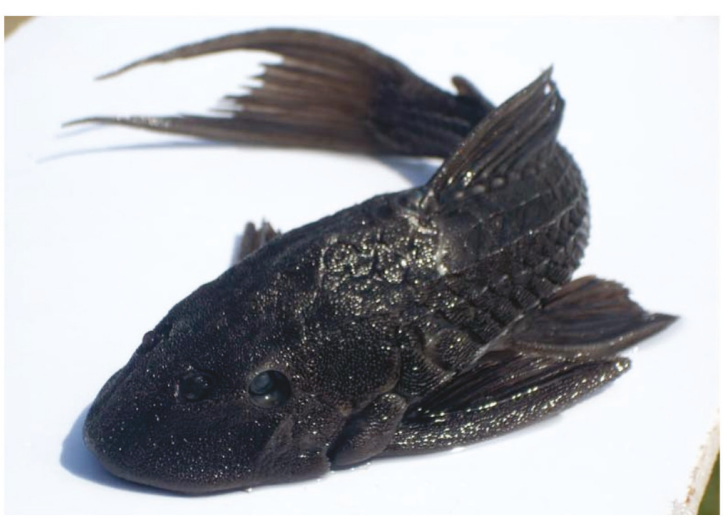

Image 33. Pseudacanthicus sp.

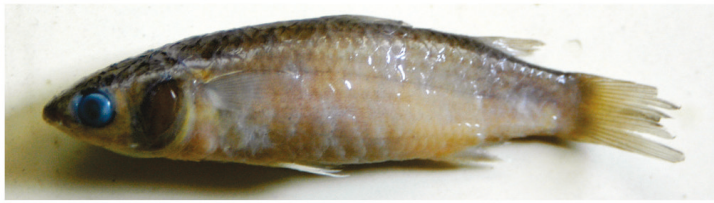

Image 35. Goldspot Mullet Chelon parsia

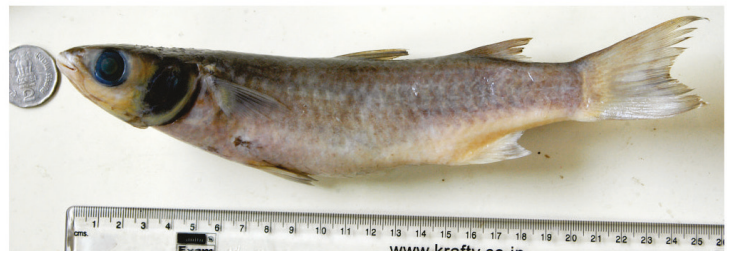

Image 37. Borneo Mullet Chelon macrolepis

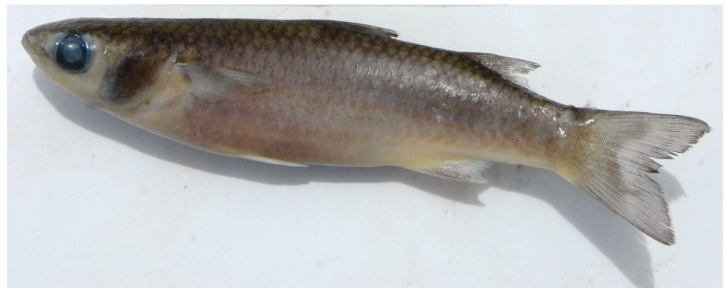

Image 39. Tade Grey Mullet Chelon planiceps

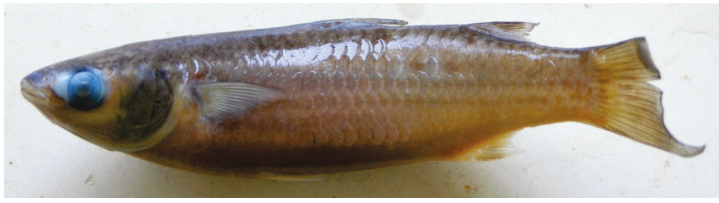

Image 41. Flathead Grey Mullet Mugil cephalus

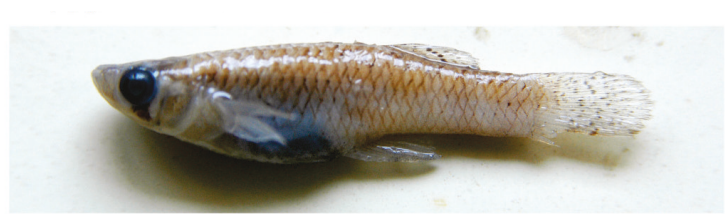

Image 43. Mosquitofish Gambusia affinis

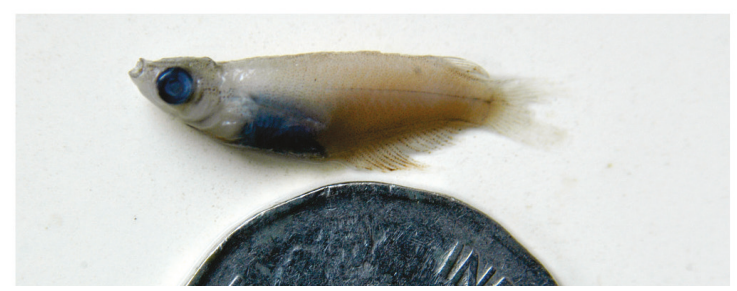

Image 34. Oryzias carnaticus

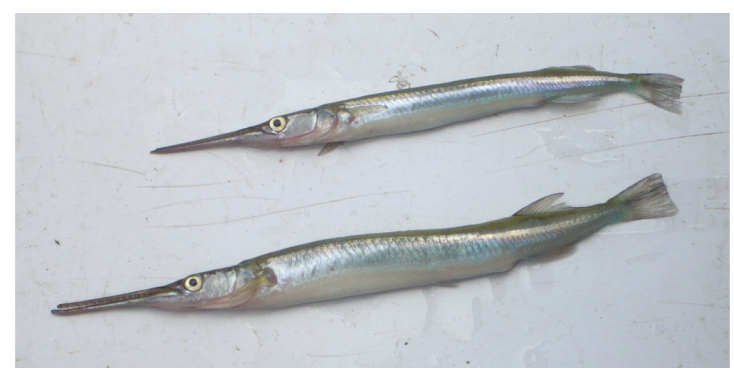

Image 36. Freshwater Garfish Xenentodon cancila

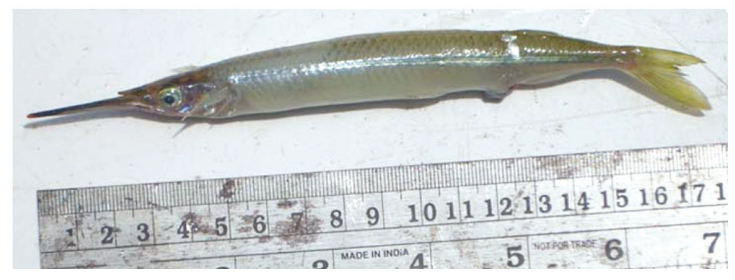

Image 38. Congaturi Halfbeak Hyporhamphus limbatus

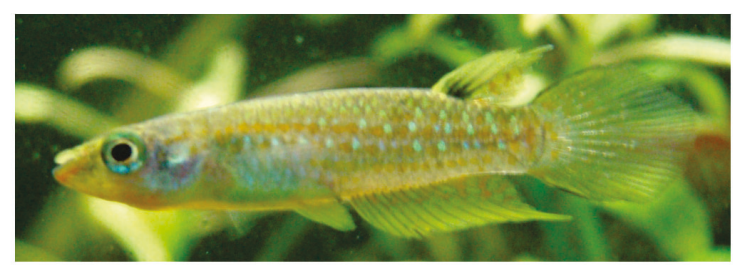

Image 40. Dwarf Panchax Aplocheilus parvus

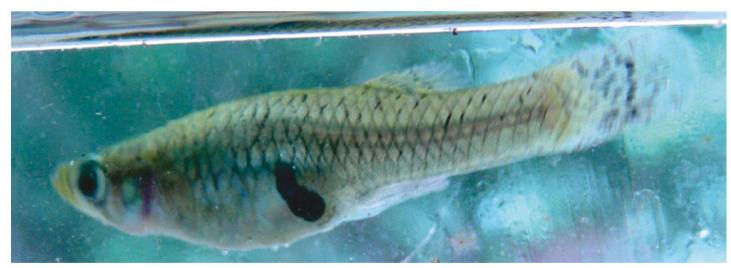

Image 42. Guppy Poecilia reticulate

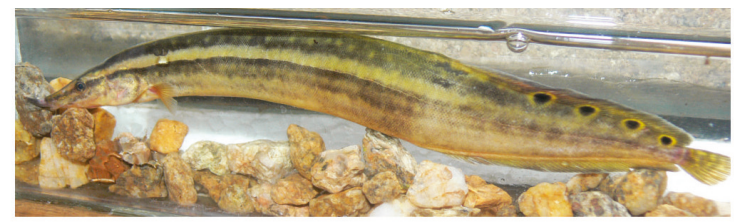

Image 44. Onestripe Spiny Eel Macrognathus aral 


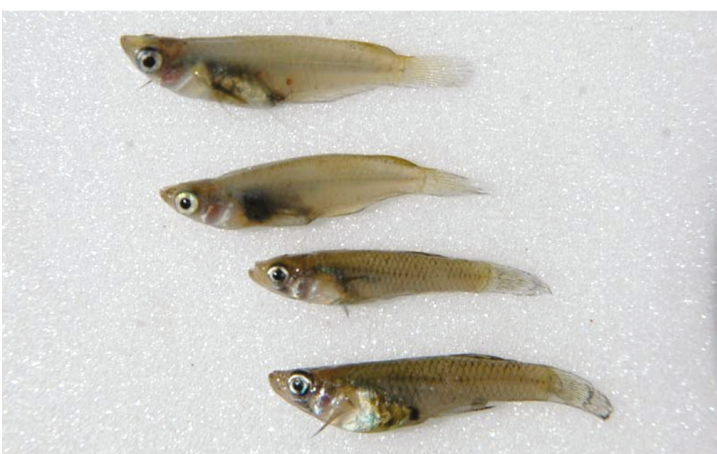

Image 45. Ricefish Oryzias dancena

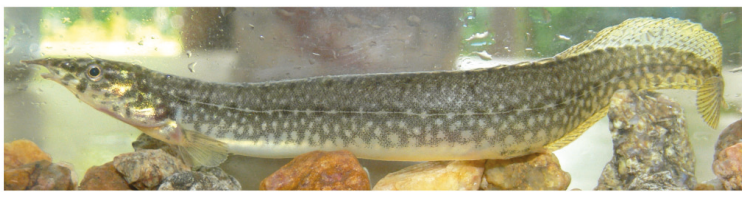

Image 47. Striped Spiny Eel Macrognathus pancalus

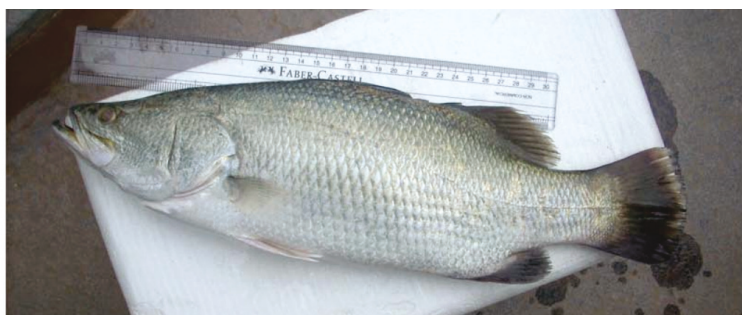

Image 49. Giant Seaperch or Seabass Lates calcarifer

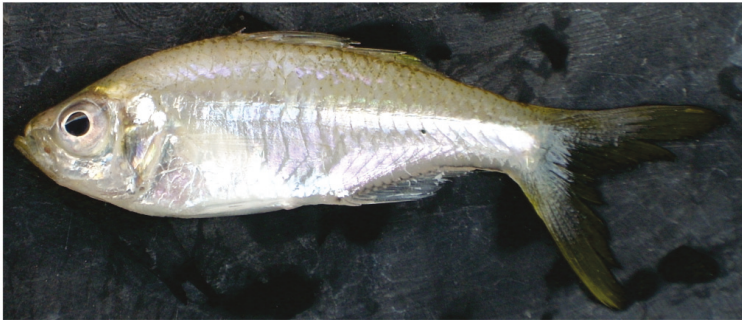

Image 51. Commerson's Glassy Perchlet Ambassis ambassis

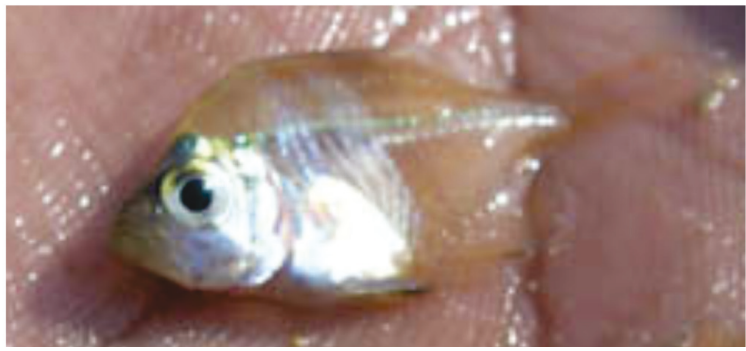

Image 53. Indian Glassy Fish Pseudambassis ranga

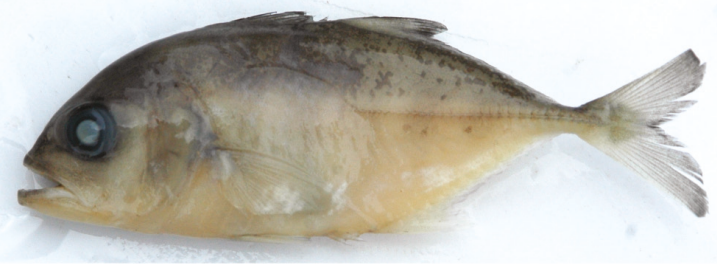

Image 46. Tille Trevally Caranx tille

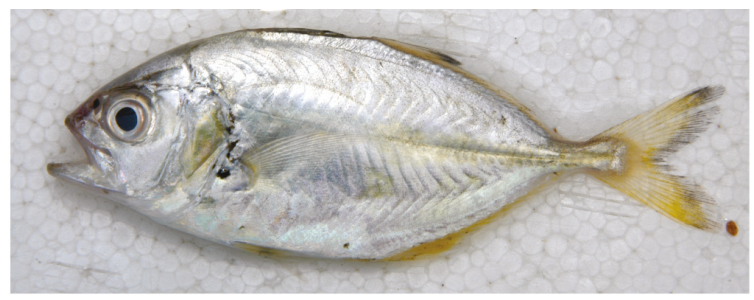

Image 48. Banded Scad Alepes kleinii

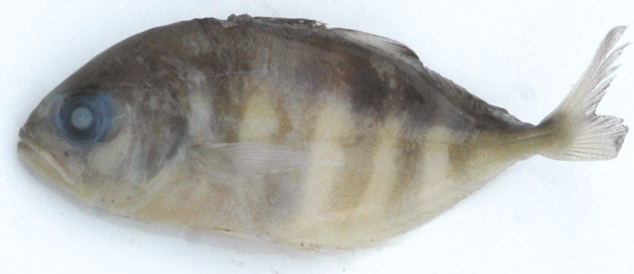

Image 50. Whitemouth Jack Uraspis helvola

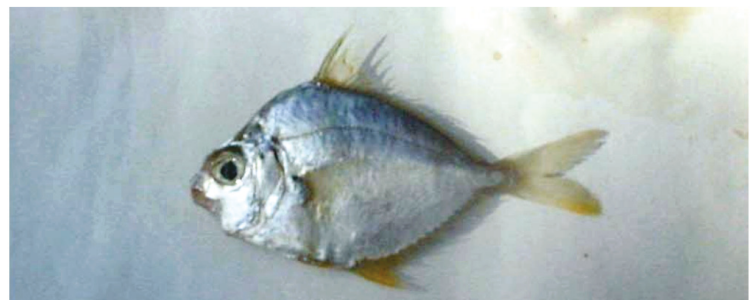

Image 52. Splendid Ponyfish Leiognathus splendens

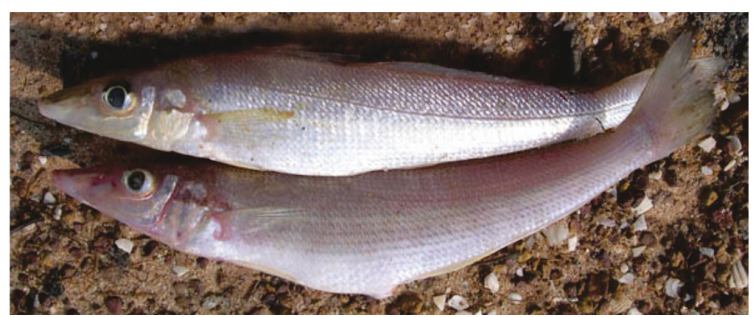

Image 54. Silver Sillago Sillago sihama

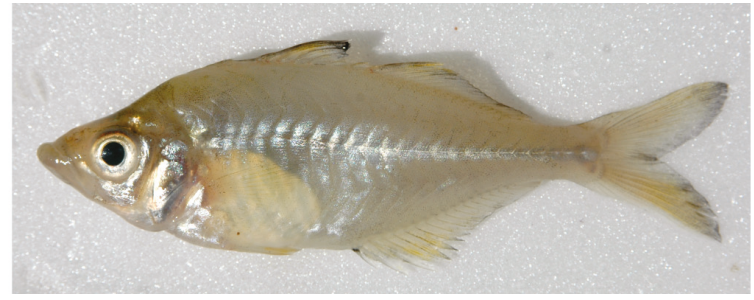

Image 55. Elongate Glass Fish Chanda nama 


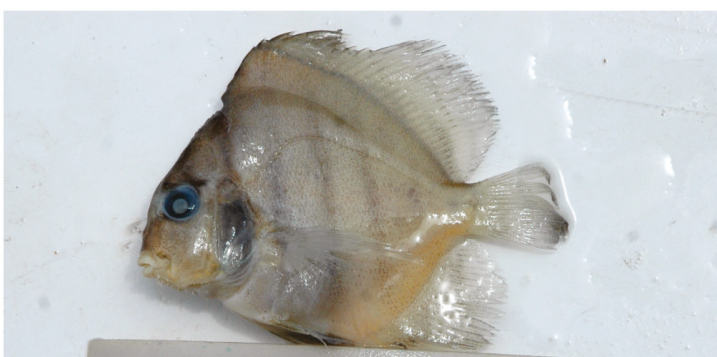

Image 56. Banded Drepane Drepane longimana

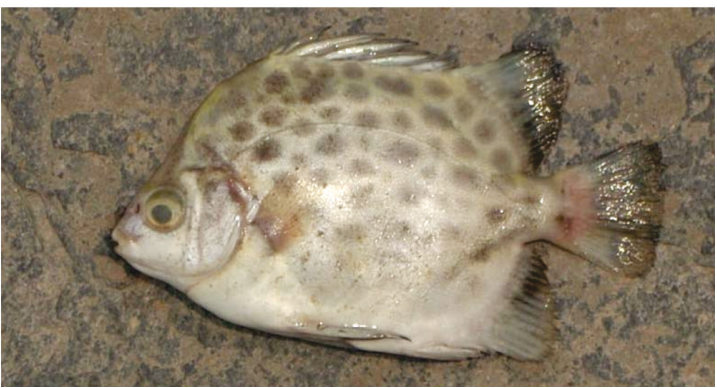

Image 58. Spotted Scat Scatophagus argus

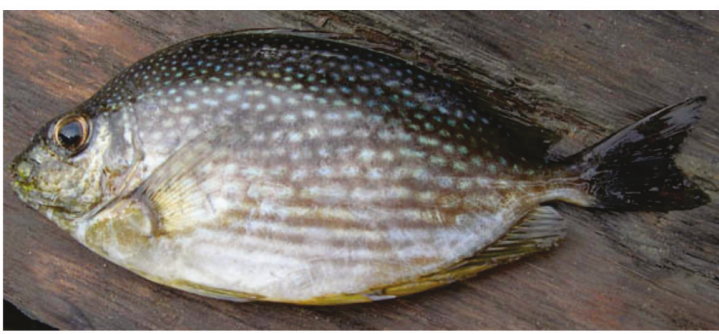

Image 60. Streaky Spinefoot Siganus javus

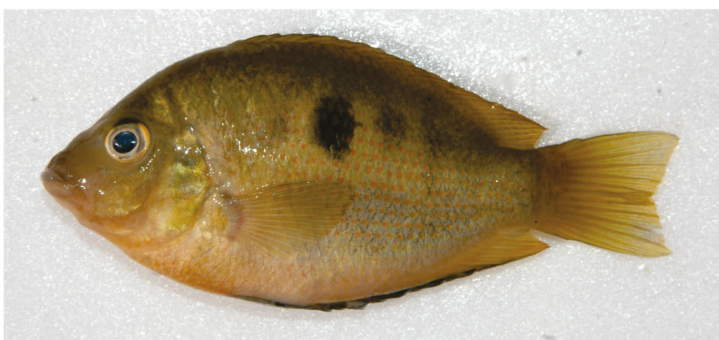

Image 63. Orange Chromide Etroplus maculatus

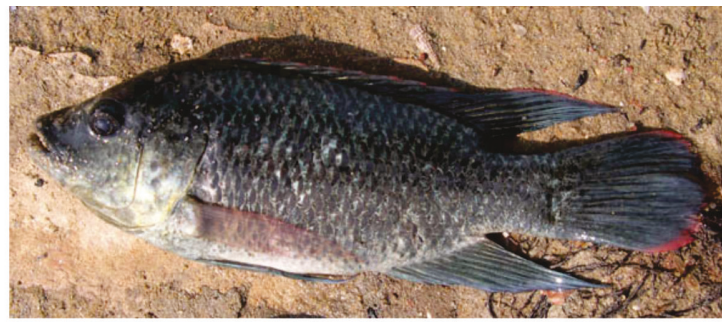

Image 65. Tilapia or Egyptian Mouthbreeder Oreochromis mossambica

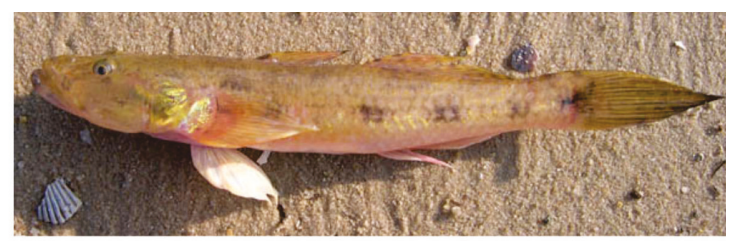

Image 57. Tank Goby Glossogobius giurus

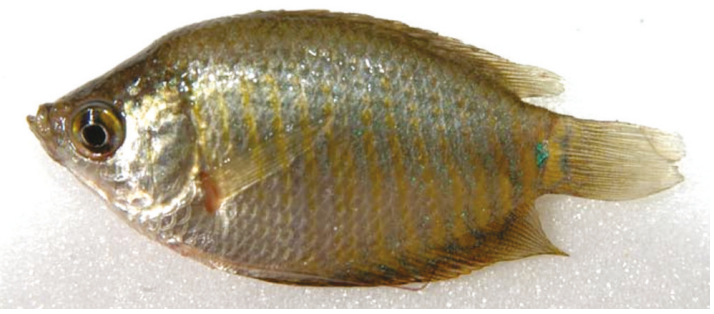

Image 59. Dwarf Gouramy Colisa Ialia

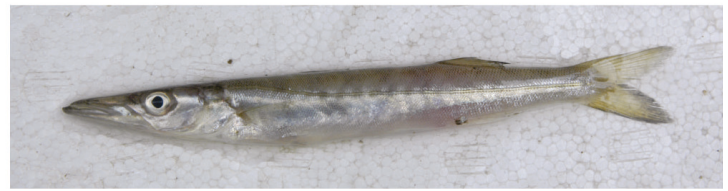

Image 61. Banded Barracuda Sphyraena jello

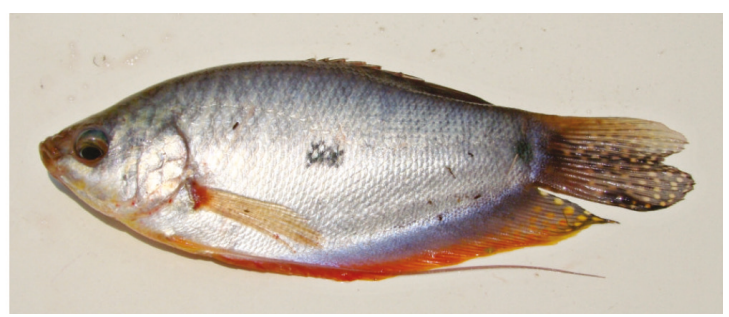

Image 62. Threespot Gouramy Trichogaster trichopterus

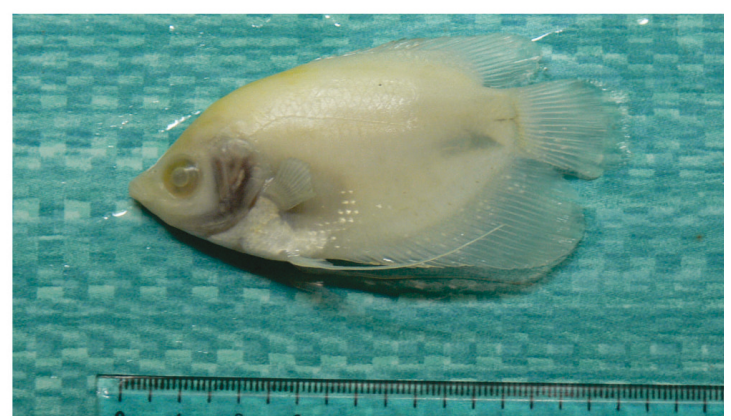

Image 64. Giant Gourami Osphronemus goramy

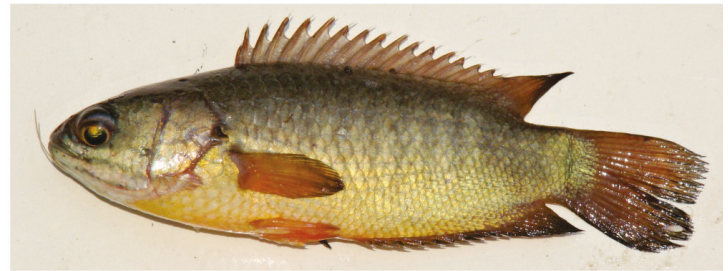

Image 66. Climbing Perch Anabas testudineus 


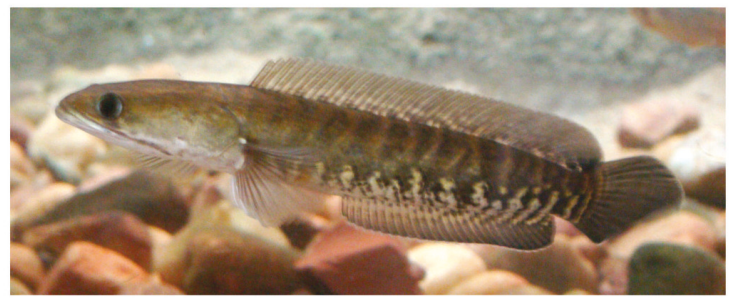

Image 67. Spotted Snakehead Channa punctatus

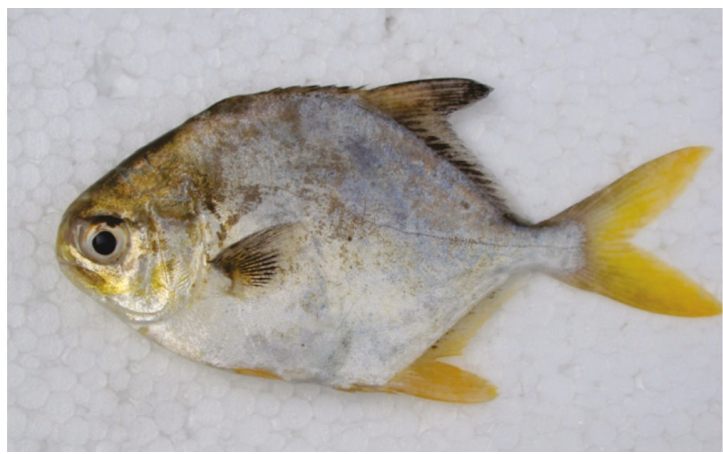

Image 69. Silver Pomfret Pampus argenteus

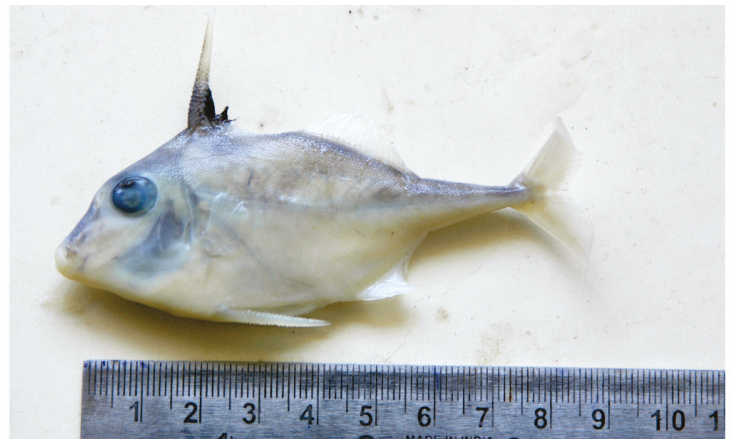

Image 71. Short-nosed Tripod Fish Triacanthus biaculeatus Image 71. Short-nosed Tripod Fish Triacanthus biaculeatus Wile:

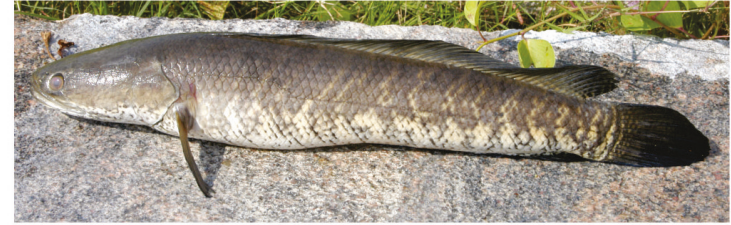

Image 68. Striped Snakehead Channa striatus

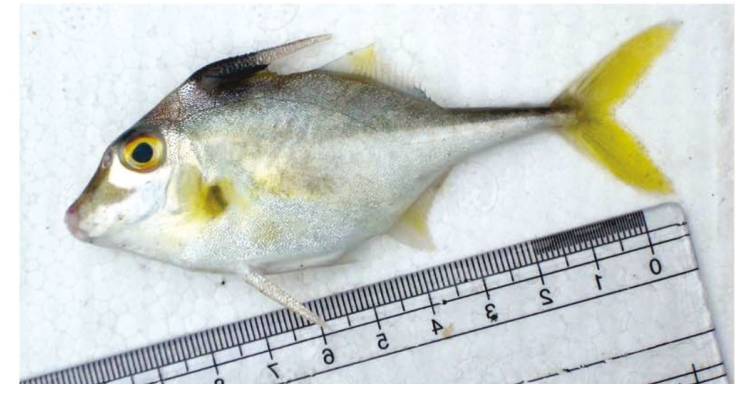

Image 70. Tripod Fish Triacanthus brevirostris 